\title{
Neutrosophic Hypersoft Matrices with Application to Solve Multiattributive Decision-Making Problems
}

\author{
Rana Muhammad Zulqarnain $\left(D,{ }^{1}\right.$ Imran Siddique, ${ }^{2}$ Rifaqat Ali $\left(\mathbb{D},{ }^{3}\right.$ Fahd Jarad $\left(D,{ }^{4,5}\right.$ \\ Abdul Samad $\mathbb{D}{ }^{6}{ }^{6}$ and Thabet Abdeljawad $\mathbb{D}^{7,8}$ \\ ${ }^{1}$ Department of Mathematics, University of Management and Technology, Lahore, Sialkot Campus, Pakistan \\ ${ }^{2}$ Department of Mathematics, University of Management and Technology, Lahore 54770, Pakistan \\ ${ }^{3}$ Department of Mathematics, College of Science and Arts, King Khalid University, Muhayil, 61413 Abha, Saudi Arabia \\ ${ }^{4}$ Department of Mathematics, Cankaya University, Etimesgut, Ankara, Turkey \\ ${ }^{5}$ Department of Medical Research, China Medical University Hospital, China Medical University, Taichung, Taiwan \\ ${ }^{6}$ School of Mathematics, Northwest University, Xi'an 710127, China \\ ${ }^{7}$ Department of Mathematics and General Sciences, Prince Sultan University, Riyadh, Saudi Arabia \\ ${ }^{8}$ Department of Computer Science and Information Engineering, Asia University, Taichung, Taiwan
}

Correspondence should be addressed to Fahd Jarad; fahd@cankaya.edu.tr and Thabet Abdeljawad; tabdeljawad@psu.edu.sa

Received 26 March 2021; Accepted 22 April 2021; Published 7 June 2021

Academic Editor: Ahmed Mostafa Khalil

Copyright (c) 2021 Rana Muhammad Zulqarnain et al. This is an open access article distributed under the Creative Commons Attribution License, which permits unrestricted use, distribution, and reproduction in any medium, provided the original work is properly cited.

\begin{abstract}
The concept of the neutrosophic hypersoft set (NHSS) is a parameterized family that deals with the subattributes of the parameters and is a proper extension of the neutrosophic soft set to accurately assess the deficiencies, anxiety, and uncertainty in decisionmaking. Compared with existing research, NHSS can accommodate more uncertainty, which is the most significant technique for describing fuzzy information in the decision-making process. The main objective of the follow-up study is to develop the theory of neutrosophic hypersoft matrix (NHSM). The NHSM is the generalized form of a neutrosophic soft matrix (NSM). Some fundamental operations and score function for NHSMs have been introduced with their desirable properties. Furthermore, we introduce the logical operators such as OR-operator and AND-operator with their fundamental properties in the following research. The necessity and possibility operations for NHSMs have been established. Utilizing the developed score function, a decision-making methodology has been developed to solve the multiattribute decision-making (MADM) problem. To ensure the validity of the proposed approach, a numerical illustration has been described for the selection of competent faculty member. The practicality and effectiveness of the current approach are proved through comparative analysis with the assistance of some existing studies.
\end{abstract}

\section{Introduction}

Decision-making (DM) is one of the most interesting problems today, choosing the right alternative for any particular purpose. Primarily, it was supposed that evidence about possible choices was collected in crisp numbers, but in real life, collective facts and data are always composed of inappropriate and erroneous information. A fuzzy set is like a set whose components have a degree of membership (Mem). In the classical set theory, the Mem degree of the elements in the set is patterned in binary form conferring to the bivalent condition of whether the elements belong to the set entirely. In distinction, the fuzzy set theory permits contemporary assessments of the remembrance of elements in the set. This is signified by the Mem function, and the actual unit interval of the Mem function is $[0,1]$. The fuzzy set is a simplification of the classic set since the indicator function of the classic set is a special case of the Mem function of the fuzzy set (if the latter only takes the value 0 or 1). In the fuzzy set theory, the classical bivalent set is generally named the crisp set. Fuzzy set theory can be used in a wide range of fields with imperfect or vague information. 
In some situations, decision-makers consider the Mem and nonmembership (Nmem) values of objects. In such cases, Zadeh's FS is unable to handle the imprecise and vague information. Atanassov [2] developed the notion of intuitionistic fuzzy sets (IFSs) to deal with the abovementioned difficulties. The IFS accommodates the imprecise and inaccurate information using Mem and Nmem values.

Atanassov's IFS is unable to solve those problems in which decision-makers considered the membership degree (MD) and nonmembership degree (NMD) such as $\mathrm{MD}=0.5$ and $\mathrm{NDM}=0.8$, then $0.5+0.8 \not 1$. Yager $[3,4]$ extended the notion of IFS to Pythagorean fuzzy sets (PFSs) to overcome the abovediscussed complications by modifying $\mathrm{MD}+\mathrm{NMD} \leq 1$ to $\mathrm{MD}^{2}+\mathrm{NMD}^{2} \leq 1$. After the development of PFSs, Zhang and $\mathrm{Xu}$ [5] proposed operational laws for PFSs and established a DM approach to resolve the MCDM problem. Wei and Lu [6] planned some power aggregation operators (AOs) and proposed a DM technique to solve multiattribute decision-making (MADM) problems under the Pythagorean fuzzy environment. Wang and Li [7] presented power Bonferroni mean operators for PFSs with their basic properties using interaction. Gao et al. [8] presented several aggregation operators by considering the interaction and proposed a DM approach to solving MADM difficulties by utilizing the developed operators. Wei [9] developed the interaction operational laws for Pythagorean fuzzy numbers (PFNs) by considering interaction and established interaction aggregation operators by using the developed interaction operations. Zhang [10] developed the accuracy function and presented a DM approach to solving multiple criteria group decision-making (MCGDM) problems using PFNs. Wang et al. [11] extended the PFSs and introduced interactive Hamacher operation with some novel AOs. They also established a DM method to solve MADM problems by using their proposed operators. Wang and $\mathrm{Li}$ [12] developed some operators for interval-valued PFSs and utilized their operators to resolve multiattribute group decision-making (MAGDM) problems. Peng and Yuan [13] established some novel operators such as Pythagorean fuzzy point operators and developed a DM technique using their proposed operators. Peng and Yang [14] introduced some operations with their desirable properties under PFSs and planned DM methodology to solve the MAGDM problem. Garg [15] developed the logarithmic operational laws for PFSs and proposed some AOs. Arora and Garg [16] presented the operational laws for linguistic IFS and developed prioritized AOs. Ma and Xu [17] presented some innovative AOs for PFSs and proposed the score and accuracy functions for PFNs.

The abovementioned theories and their DM methodologies have been used in several fields of life, but these theories are unable to deal with the parametrization of the alternatives. Molodtsov [18] developed the soft sets (SSs) to overcome the abovementioned complications. Molodtsov's SS competently deals with imprecise, vague, and unclear information of objects considering their parametrization. Maji et al. [19] prolonged the concept of SS and introduced basic operations with their properties. Maji et al. [20] established a DM technique using their developed operations for SS. They also merged two well-known theories such as FS and SS and established the concept of fuzzy soft sets (FSSs) [21]. They also proposed the notion of an intuitionistic fuzzy soft set (IFSS) [22] and discussed their basic operations. Garg and Arora [23] extended the notion of IFSS and presented a generalized form of IFSS with AOs. They also planned a DM technique to resolve undefined and inaccurate information under IFSS information. Garg and Arora [24] presented the correlation and weighted correlation coefficients for IFSS and extended the TOPSIS technique using developed correlation measures. Zulqarnain et al. [25] introduced some AOs and correlation coefficients for interval-valued IFSS. They also extended the TOPSIS technique using their developed correlation measures and utilized them to solve the MADM problem. Peng et al. [26] proposed the Pythagorean fuzzy soft sets (PFSSs) and presented fundamental operations of PFSSs with their desirable properties by merging PFS and SS. Athira et al. [27] extended the notion of PFSSs and proposed entropy measures for PFSSs. They also presented some distance measures for PFSSs and utilized their developed distance measures to solve DM [28] issues. Zulqarnain et al. [29] introduced operational laws for Pythagorean fuzzy soft numbers (PFSNs) and developed AOs such as Pythagorean fuzzy soft weighted average and geometric by using defined operational laws for PFSNs. They also planned a DM approach to solve MADM problems with the help of presented operators. Riaz et al. [30] prolonged the idea of PFSSs and developed the $m$ polar PFSSs. They also established the TOPSIS method under the considered hybrid structure and proposed a DM methodology to solve the MCGDM problem. Riaz et al. [31] developed the similarity measures for PFSS with their fundamental properties. Han et al. [32] protracted the TOPSIS method under PFSS environment and utilized their developed approach to solving the MAGDM problem. Zulqarnain et al. [33] planned the TOPSIS methodology in the PFSS environment based on the correlation coefficient. They also established a DM methodology to resolve the MCGDM concerns and utilized the developed approach in green supply chain management.

All the above studies only deal the inadequate information because of membership and nonmembership values; however, these theories cannot handle the overall incompatible and imprecise information. To address such incompatible and imprecise records, the idea of the neutrosophic set (NS) was developed by Smarandache [34]. Maji [35] offered the concept of a neutrosophic soft set (NSS) with necessary operations and properties. The idea of the possibility NSS was developed by Karaaslan [36] and introduced a possibility of neutrosophic soft DM method to solve those problems which contain uncertainty based on AND-product. Broumi [37] developed the generalized NSS with some operations and properties and used the projected concept for DM. Deli and Subas [38] developed the singlevalued neutrosophic numbers (SVNNs) to solve MCDM problems. They also established the cut sets for SVNNs. Wang et al. [39] proposed the correlation coefficient (CC) for SVNSs. Ye [40] introduced the simplified NSs with some operational laws and AOs such as weighted arithmetic and 
weighted geometric average operators and constructed an MCDM method based on his proposed AOs. Masooma et al. [41] progressed a new concept through combining the multipolar fuzzy set and NS which is known as the multipolar NS, and they also established various characterization and operations with examples. Zulqarnain et al. [42, 43] presented the generalized neutrosophic TOPSIS and an integrated model of neutrosophic TOPSIS, respectively, and used their presented technique for supplier selection in the production industry.

All the abovementioned studies have some limitations, such as when any attribute from a set of attributes contains further subattribute, and then the abovepresented theories fail to solve such types of problems. To overcome the aforementioned limitations, Smarandache [44] protracted the idea of SS to hypersoft sets (HSSs) by substituting the one-parameter function $f$ to a multiparameter (subattribute) function. Samarandache claimed that the established HSS is competently dealing with uncertain objects comparative to SS. Nowadays, HSS theory and its extensions have been arising unexpectedly. Several investigators go through progressed distinctive operators along with characteristics under HSS and its extensions [45, 46]. Zulqarnain et al. [47] presented the IFHSS which is the generalized version of IFSS. They established the TOPSIS method to resolve the MADM problem utilizing the developed correlation coefficient. Zulqarnain et al. [48] introduced the notion of the Pythagorean fuzzy hypersoft set (PFHSS) with some fundamental operations and discussed their desirable properties. They also proposed the CC for PFHSS and established a decision-making approach to solve decision-making complications utilizing their developed CC. Zulqarnain et al. [49] proposed the AOs for PFHSS and correlation coefficients. They also established the TOPSIS technique using their developed correlation coefficient and utilized the presented approach for the selection of appropriate antivirus face masks because the above work is considered to examine the environment of linear inequality between the MD and NMD of subattributes of the considered attributes. Samad et al. [50] extended the TOPSIS technique under NHSS based on $\mathrm{CC}$ and utilized their presented decision-making technique to solve the MADM problem. Zulqarnain et al. [51, 52] extended the NHSS to interval-valued NHSS with some fundamental operations and a decision-making technique to solve DM issues utilizing their developed CC.

Matrices perform a significant role in a wide area of science, engineering, and medical. Cagman et al. [53] presented soft matrices and applied them to the decisionmaking issue. They also presented fuzzy soft matrices [54] with fundamental operations and discussed their desirable properties. Furthermore, Saikia et al. [55] characterized fuzzy soft matrices with four unique results of generalized intuitionistic fuzzy soft matrices and introduced an application in medical diagnosis. Next, Broumi et al. [56] contemplated a fuzzy soft matrix dependent on reference work and characterized some new operations, for example, fuzzy soft compliment matrix, a trace of fuzzy soft matrix dependent on reference work. Another fuzzy soft matrix decision technique dependent on reference work is exhibited.
Mondal et al. [57] presented a fuzzy and intuitionistic fuzzy soft matrix and multicriteria decision-making dependent on three fundamental t-norms operators. Kalaichelvi and Kanimozhi [58] presented the intuitionistic fuzzy soft matrices by extending the fuzzy soft matrices. Since uncertainty helps in the detailing of genuine logical numerical problems, we tackle uncertainty with multiattribute decision-making (MADM) problems. In MADM problems, we have different attributes and we select the perfect match but if we have a complex selection like multiargument problems, then we use the concept of hypersoft set. It is observed that fuzzy numbers can only measure uncertainty and intuitionistic fuzzy numbers can measure the truth and falsity membership values. But if we want to measure the membership values of truth, indeterminacy, and falsity, we need to work in the neutrosophic environment because it is more reliable, logical, and practical for the decision-makers. Dealing with neutrosophic hypersoft set is difficult because of its complicated framework. From this aspect, we will attempt to convert neutrosophic hypersoft set to neutrosophic hypersoft matrix in this article.

The rest of the article can be summarized like this. In Section 2, we presented some basic notions such as soft set, neutrosophic soft set, hypersoft set, and neutrosophic hypersoft sets, which are used to develop the structure of the current research. In Section 3, we planned the NHSM with some fundamental operations and score function with their desirable properties. Also, we presented some logical operations, necessity, and possibility operators for NHSM with their properties in Section 4. To solve the MADM problem, an algorithm is established by using the presented score function provided in Section 5. Moreover, the planned DM method is used for the selection of the most suitable faculty position. Also, we apply some available techniques to propose a comparative analysis of our planned approach in Section 6.

\section{Preliminaries}

In the following section, we recalled fundamental concepts that help us to develop the structure of the current article such as SS, NS, NSS, HSS, and NHSS.

Definition 1 (see [18]). Let $\mathcal{U}$ be the universal set and $\mathscr{E}$ be the set of attributes with respect to $\mathcal{U}$. Let $(\mathcal{U})$ be the power set of $\mathcal{U}$ and $\mathscr{A} \subseteq \mathscr{E}$. A pair $(\mathscr{F}, \mathscr{A})$ is called a soft set over $\mathscr{U}$, and its mapping is given as

$$
\mathscr{F}: \mathscr{A} \longrightarrow P(\mathscr{U}) \text {. }
$$

It is also defined as

$$
(\mathscr{F}, \mathscr{A})=\{\mathscr{F}(e) \in P(\mathscr{U}): e \in \mathscr{E}, \mathscr{F}(e)=\varnothing, \text { if } e \notin \mathscr{A}\} .
$$

Definition 2 (see [34]). Let $\mathscr{U}$ be a universe and $\mathscr{A}$ be an NS on $\mathscr{U}$ defined as $\mathscr{A}=\left\{v,\left(\mathscr{T}_{\mathscr{A}}(v), \mathscr{I}_{\mathscr{A}}(v), \mathfrak{C}_{\mathscr{A}}(v)\right): v \in \mathscr{U}\right\}$, where $\mathscr{T}, \mathscr{I}, \quad \mathfrak{S}: \mathscr{U} \longrightarrow] 0^{-}, 1^{+}\left[\right.$, and $0^{-} \leq \mathscr{T}_{\mathscr{A}}(v)+$ $\mathscr{I}_{\mathscr{A}}(v)+\mathfrak{C}_{\mathscr{A}}(v) \leq 3^{+}$. 
Definition 3 (see [35]). Let $\mathcal{U}$ be the universal set and $\mathscr{E}$ be the set of attributes with respect to $\mathcal{U}$. Let $(\mathcal{U})$ be the set of neutrosophic values of $\mathscr{U}$ and $\mathscr{A} \subseteq \mathscr{E}$. A pair $(\mathscr{F}, \mathscr{A})$ is called a neutrosophic soft set over $\mathcal{U}$, and its mapping is given as

$$
\mathscr{F}: \mathscr{A} \longrightarrow P(\mathscr{U}) \text {. }
$$

Definition 4 (see [43]). Let $\mathcal{U}$ be the universal set and $(\mathcal{U})$ be the power set of $\mathcal{U}$. Consider $\ell^{1}, \ell^{2}, \ell^{3}, \ldots, \ell^{n}$ for $n \geq 1$ be $n$ well-defined attributes, whose corresponding attributive values are, respectively, the set $\mathscr{L}^{1}, \mathscr{L}^{2}, \mathscr{L}^{3}, \ldots, \mathscr{L}^{n}$ with $\mathscr{L}^{i} \cap \mathscr{L}^{j}=\varnothing$, for $i \neq j$ and $i, j \mathcal{E}\{1,2,3, \ldots, n\}$, then the pair $\left(\mathscr{F}, \mathscr{L}^{1} \times \mathscr{L}^{2} \times \mathscr{L}^{3}, \ldots, \mathscr{L}^{n}\right)$ is said to be hypersoft set over $\mathcal{U}$, where

$$
\mathscr{F}: \mathscr{L}^{1} \times \mathscr{L}^{2} \times \mathscr{L}^{3}, \ldots, \mathscr{L}^{n} \longrightarrow P(\mathscr{U}) .
$$

TABLE 1: Tabular representation of the characteristic function.

\begin{tabular}{ccccc}
\hline & $\mathscr{L}_{1}^{a}$ & $\mathscr{L}_{2}^{b}$ & $\ldots$ & $\mathscr{L}_{\beta}^{z}$ \\
\hline $\mathbf{u}^{1}$ & $\mathscr{X}_{\mathscr{R}_{\mathfrak{Q}}}\left(u^{1}, \mathscr{L}_{1}^{a}\right)$ & $\mathscr{X}_{\mathscr{R}_{\mathfrak{Q}}}\left(u^{1}, \mathscr{L}_{2}^{b}\right)$ & $\ldots$ & $\mathscr{X}_{\mathscr{R}_{\mathfrak{R}}}\left(u^{1}, \mathscr{L}_{\beta}^{z}\right)$ \\
$\mathbf{u}^{2}$ & $\mathscr{X}_{\mathscr{R}_{\mathfrak{Q}}}\left(u^{2}, \mathscr{L}_{1}^{a}\right)$ & $\mathscr{X}_{\mathscr{R}_{\mathfrak{Q}}}\left(u^{2}, \mathscr{L}_{2}^{b}\right)$ & $\ldots$ & $\mathscr{X}_{\mathscr{R}_{\mathfrak{Q}}}\left(u^{2}, \mathscr{L}_{\beta}^{z}\right)$ \\
$\vdots$ & $\vdots$ & $\vdots$ & $\ddots$ & $\vdots$ \\
$\mathbf{u}^{\alpha}$ & $\mathscr{X}_{\mathscr{R}_{\Omega}}\left(u^{\alpha}, \mathscr{L}_{1}^{a}\right)$ & $\mathscr{X}_{\mathscr{R}_{\Omega}}\left(u^{\alpha}, \mathscr{L}_{2}^{b}\right)$ & $\ldots$ & $\mathscr{X}_{\mathscr{R}_{\mathfrak{Q}}}\left(u^{\alpha}, \mathscr{L}_{\beta}^{z}\right)$ \\
\hline
\end{tabular}

Definition 5 (see [43]). Let $\mathcal{U}$ be the universal set and $(\mathcal{U})$ be the power set of $\mathscr{E}$. Consider $\ell^{1}, \ell^{2}, \ell^{3}, \ldots, \ell^{n}$ for $n \geq 1$, be $n$ well-defined attributes, whose corresponding attributive values are, respectively, the set $\mathscr{L}^{1}, \mathscr{L}^{2}, \mathscr{L}^{3}, \ldots, \mathscr{L}^{n}$ with $\mathscr{L}^{i} \cap \mathscr{L}^{j}=\varnothing$, for $i \neq j$ and $i, j \varepsilon\{1,2,3, \ldots, n\}$ and their relation $\mathscr{L}^{1}, \mathscr{L}^{2}, \mathscr{L}^{3}, \ldots, \mathscr{L}^{n}=\mathcal{S}$, then the pair $(\mathscr{F}, \mathcal{S})$ is said to be neutrosophic hypersoft set (NHSS) over $\mathscr{U}$, where

$$
\begin{aligned}
& \mathscr{F}: \mathscr{L}^{1}, \mathscr{L}^{2}, \mathscr{L}^{3}, \ldots, \mathscr{L}^{n} \longrightarrow P(\mathscr{U}), \\
& \mathscr{F}\left(\mathscr{L}^{1}, \mathscr{L}^{2}, \mathscr{L}^{3}, \ldots, \mathscr{L}^{n}\right)=\{x, \mathscr{T}(\mathscr{F}(\mathcal{S})), \mathscr{I}(\mathscr{F}(\mathcal{S})), \mathscr{F}(\mathscr{F}(\mathcal{S})), x \in \mathscr{U}\},
\end{aligned}
$$

where $\mathscr{T}$ is the membership value of truthiness, $\mathscr{I}$ is the membership value of indeterminacy, and $\mathscr{F}$ is the membership value of falsity such that $\mathscr{T}, \mathscr{J}, \mathscr{F}: \mathcal{U} \longrightarrow[0,1]$ also $0 \leq \mathscr{T}(\mathscr{F}(\mathcal{S}))+\mathscr{I}(\mathscr{F}(\mathcal{S}))+\mathscr{F}(\mathscr{F}(\mathcal{S})) \leq 3$.

\section{Development of Neutrosophic Hypersoft Matrix with Their Fundamental Operators}

In this section, we introduced the notion of a neutrosophic hypersoft matrix with some basic operations and discuss their desirable properties.
Definition 6 Let $\mathcal{U}=\left\{u^{1}, u^{2}, \ldots, u^{\alpha}\right\}$ and $(\mathcal{U})$ be the universal set and power set of universal set, respectively, and also consider $\mathscr{L}_{1}, \mathscr{L}_{2}, \ldots, \mathscr{L}_{\beta}$ for $\beta \geq 1, \beta$ well-defined attributes, whose corresponding attributive values are, respectively, the set $\mathscr{L}_{1}^{a}, \mathscr{L}_{2}^{b}, \ldots, \mathscr{L}_{\beta}^{z}$ and their relation $\mathscr{L}_{1}^{a} \times \mathscr{L}_{2}^{b} \times \cdots \times \mathscr{L}_{\beta}^{z}$, where $a, b, c, \ldots, z=1,2, \ldots, n$, then the pair $\left(\mathscr{F}, \mathscr{L}_{1}^{a} \times \mathscr{L}_{2}^{b} \times \cdots \times \mathscr{L}_{\beta}^{z}\right)$ is said to be neutrosophic hypersoft set over $\mathscr{U}$, where $\mathscr{F}:\left(\mathscr{L}_{1}^{a} \times \mathscr{L}_{2}^{b} \times \cdots \times \mathscr{L}_{\beta}^{z}\right)$ $\longrightarrow P(\mathcal{U})$, and it is defined as

$$
\mathscr{F}\left(\mathscr{L}_{1}^{a} \times \mathscr{L}_{2}^{b} \times \cdots \times \mathscr{L}_{\beta}^{z}\right)=\left\{u, T_{\mathfrak{Q}}(u), I_{\mathfrak{Q}}(u), F_{\mathfrak{Q}}(u): u \in \mathcal{U}, \mathfrak{Q} \in \mathscr{L}_{1}^{a} \times \mathscr{L}_{2}^{b} \times \cdots \times \mathscr{L}_{\beta}^{z}\right\}
$$

Let $\mathscr{R}_{\mathbb{Q}}=\mathscr{L}_{1}^{a} \times \mathscr{L}_{2}^{b} \times \cdots \times \mathscr{L}_{\beta}^{z}$ be the relation, and its characteristic function is $\mathscr{X}_{\mathscr{R}_{\Omega}}: \mathscr{L}_{1}^{a} \times \mathscr{L}_{2}^{b} \times \cdots \times \mathscr{L}_{\beta}^{z} \longrightarrow$ $P(\mathscr{U})$; it is defined as $\mathscr{X}_{\mathscr{R}_{\mathfrak{R}}}=\left\{u, T_{\mathfrak{Q}}(u), I_{\mathfrak{L}}(u), F_{\mathfrak{Q}}(u)\right.$ : $\left.u \in \mathscr{U}, \mathfrak{R} \in \mathscr{L}_{1}^{a} \times \mathscr{L}_{2}^{b} \times \cdots \times \mathscr{L}_{\beta}^{z}\right\}$ and can be a representation of $\mathscr{R}_{\mathfrak{Q}}$ as given in Table 1 .

If $O_{i j}=\mathscr{X}_{\mathscr{R}_{\mathfrak{g}}}\left(u^{i}, \mathscr{L}_{j}^{k}\right)$, where $i=1,2,3, \ldots, \alpha, j=1,2,3$ $, \ldots, \beta, k=a, b, c, \ldots, z$, then a matrix is defined as

$$
\left[O_{i j}\right]_{\alpha \times \beta}=\left(\begin{array}{cccc}
O_{11} & O_{12} & \cdots & O_{1 \beta} \\
O_{21} & O_{22} & \cdots & O_{2 \beta} \\
\vdots & \vdots & \ddots & \vdots \\
O_{\alpha 1} & O_{\alpha 2} & \cdots & O_{\alpha \beta}
\end{array}\right)
$$

where $O_{i j}=\left(T_{\mathscr{L}_{j}^{k}}\left(u_{i}\right), I_{\mathscr{L}_{j}^{k}}\left(u_{i}\right), F_{\mathscr{L}_{j}^{k}}\left(u_{i}\right), u_{i} \in \mathscr{U}, \mathscr{L}_{j}^{k} \in \mathscr{L}_{1}^{a} \times\right.$ $\left.\mathscr{L}_{2}^{b} \times \cdots \times \mathscr{L}_{\beta}^{z}\right)=\left(\mathscr{T}_{i j k}^{o}, \mathscr{J}_{i j k}^{o}, \mathscr{F}_{i j k}^{o}\right)$.
Thus, we can represent any neutrosophic hypersoft set in terms of neutrosophic hypersoft matrix (NHSM), and it means that they are interchangeable.

Example 1. Let $\mathcal{U}$ be the set of candidates for the teaching at the college level:

$$
\mathcal{U}=\left\{\mathscr{T}^{1}, \mathscr{T}^{2}, \mathscr{T}^{3}, \mathscr{T}^{4}, \mathscr{T}^{5}\right\}
$$

and also consider the set of attributes as

$$
\begin{aligned}
& \mathscr{A}_{1}=\text { Qualification }, \\
& \mathscr{A}_{2}=\text { Experience }, \\
& \mathscr{A}_{3}=\text { Gender } \\
& \mathscr{A}_{4}=\text { Publications } .
\end{aligned}
$$

Their respective attributes are given as 


$$
\begin{aligned}
& \mathscr{A}_{1}^{a}=\text { Qualification }=\left\{\text { BS Hons. }, \frac{\mathrm{MS}}{\mathrm{Mphill}}, \text { Phd, Post Doctorate }\right\}, \\
& \mathscr{A}_{2}^{b}=\text { Experience }=\{5 \mathrm{yr}, 8 \mathrm{yr}, 10 \mathrm{yr}, 15 \mathrm{yr}\}, \\
& \mathscr{A}_{3}^{c}=\text { Gender }=\{\text { Male, } \text { Female }\} \\
& \mathscr{A}_{4}^{d}=\text { Publications }=\{3,5,8,10+\} .
\end{aligned}
$$

Let the function be $\mathscr{F}: \mathscr{A}_{1}^{a} \times \mathscr{A}_{2}^{b} \times \mathscr{A}_{3}^{c} \times \mathscr{A}_{4}^{d} \longrightarrow P(\mathscr{U})$.
The tabular representation is given in Tables $2-5$ with their neutrosophic values from different decision-makers.

Neutrosophic hypersoft set is defined as

$$
\mathscr{F}:\left(\mathscr{A}_{1}^{a} \times \mathscr{A}_{2}^{b} \times \mathscr{A}_{3}^{c} \times \mathscr{A}_{4}^{d}\right) \longrightarrow P(\mathscr{U}) .
$$

Let us assume $\mathscr{F}\left(\mathscr{A}_{1}^{a} \times \mathscr{A}_{2}^{b} \times \mathscr{A}_{3}^{c} \times \mathscr{A}_{4}^{d}\right)=\mathscr{F}$ (Mphill, 5 yr, male, 3) $=\left\{\mathscr{T}^{1}, \mathscr{T}^{2}, \mathscr{T}^{4}, \mathscr{T}^{5}\right\}$.

Then, the neutrosophic hypersoft set of aboveassumed relation is

$$
\begin{aligned}
\mathscr{F} & \left(\mathscr{A}_{1}^{a} \times \mathscr{A}_{2}^{b} \times \mathscr{A}_{3}^{c} \times \mathscr{A}_{4}^{d}\right) \\
= & \mathscr{F}(\text { Mphill, 5 yr, male }, 3) \\
= & \left\{\begin{array}{l}
\left(\mathscr{T}^{1},\{0.5,0.3,0.6\},\{0.3,0.4,0.7\},\{0.5,0.6,0.9\},\{0.6,0.4,0.5\}\right), \\
\left(\mathscr{T}^{2},\{0.3,0.2,0.1\},\{0.6,0.5,0.3\},\{0.7,0.8,0.3\},\{0.7,0.5,0.3\}\right), \\
\left(\mathscr{T}^{4},\{0.7,0.3,0.6\},\{0.6,0.4,0.8\},\{0.8,0.5,0.4\},\{0.6,0.2,0.1\}\right), \\
\left(\mathscr{T}^{5},\{0.5,0.4,0.5\},\{0.3,0.6,0.7\},\{0.9,0.2,0.1\},\{0.4,0.5,0.3\}\right)
\end{array}\right\} .
\end{aligned}
$$

The tabular representation of a characteristic function is given in Table 6.
Also, it can be represented in matrix form as follows:

$$
[O]_{4 \times 4}=\left[\begin{array}{cccc}
(0.5,0.3,0.6) & (0.3,0.4,0.7) & (0.5,0.6,0.9) & (0.6,0.4,0.5) \\
(0.3,0.2,0.1) & (0.6,0.5,0.3) & (0.7,0.8,0.3) & (0.7,0.5,0.3) \\
(0.7,0.3,0.6) & (0.6,0.4,0.8) & (0.8,0.5,0.4) & (0.6,0.2,0.1) \\
(0.5,0.4,0.5) & (0.3,0.6,0.7) & (0.9,0.2,0.1) & (0.4,0.5,0.3)
\end{array}\right]
$$

Definition 7 Let $O=\left[O_{i j}\right]$ be the NHSM of order $\alpha \times \beta$, where $O_{i j}=\left(\mathscr{T}_{i j k}^{o}, \mathscr{J}_{i j k}^{o}, \mathscr{F}_{i j k}^{o}\right)$, then $O$ is said to be square NHSM if $\alpha=\beta$. It means that if an NHSM has the same number of rows (attributes) and columns (alternatives), then it is a square NHSM.

Definition 8 Let $O=\left[O_{i j}\right]$ be the square NHSM of order $\alpha \times \beta$, where $O_{i j}=\left(\mathscr{T}_{i j k}^{o}, \mathscr{I}_{i j k}^{o}, \mathscr{F}_{i j k}^{o}\right)$, then $O^{t}$ is said to be the transpose of square NHSM if rows and columns of $O$ are interchanged. It is denoted as

$$
O^{t}=\left[O_{i j}\right]^{t}=\left(\mathscr{T}_{i j k}^{o}, \mathscr{J}_{i j k}^{o}, \mathscr{F}_{i j k}^{o}\right)^{t}=\left(\mathscr{T}_{j k i}^{o}, \mathscr{J}_{j k i}^{o}, \mathscr{F}_{j k i}^{o}\right)=\left[O_{j i}\right] .
$$

Definition 9 Let $O=\left[O_{i j}\right]$ be the square NHSM of order $\alpha \times \beta$, where $O_{i j}=\left(\mathscr{T}_{i j k}^{o}, \mathscr{J}_{i j k}^{o}, \mathscr{F}_{i j k}^{o}\right)$, then $O$ is said to be symmetric NHSM if $O^{t}=O$, i.e., $\left(\mathscr{T}_{i j k}^{o}, \mathscr{J}_{i j k}^{o}, \mathscr{F}_{i j k}^{o}\right)=\left(\mathscr{T}_{j k i}^{o}, \mathscr{J}_{j k i}^{o}, \mathscr{F}_{j k i}^{o}\right)$.

Definition 10 Let $O=\left[O_{i j}\right]$ be the NHSM of order $\alpha \times \beta$, where $O_{i j}=\left(\mathscr{T}_{i j k}^{o}, \mathscr{J}_{i j k}^{o}, \mathscr{F}_{i j k}^{o}\right)$ and $s$ be any scalar, then the product of matrix $O$ and a scalar $s$ is a matrix formed by multiplying each element of matrix $O$ by $s$. It is denoted as $s \mathrm{O}=\left[s \mathrm{~S}_{i j}\right]$, where $0 \leq s \leq 1$.

Example 2. Let us consider an NHSM $[O]_{4 \times 4}$.

$$
[O]_{4 \times 4}=\left[\begin{array}{cccc}
(0.5,0.3,0.6) & (0.3,0.4,0.7) & (0.5,0.6,0.9) & (0.6,0.4,0.5) \\
(0.3,0.2,0.1) & (0.6,0.5,0.3) & (0.7,0.8,0.3) & (0.7,0.5,0.3) \\
(0.7,0.3,0.6) & (0.6,0.4,0.8) & (0.8,0.5,0.4) & (0.6,0.2,0.1) \\
(0.5,0.4,0.5) & (0.3,0.6,0.7) & (0.9,0.2,0.1) & (0.4,0.5,0.3)
\end{array}\right],
$$


TABle 2: Opinion of decision-makers for all alternatives against $\mathscr{A}_{1}^{a}$.

\begin{tabular}{|c|c|c|c|c|c|}
\hline $\mathscr{A}_{1}^{a}$ (Qualification) & $\mathscr{T}^{1}$ & $\mathscr{T}^{2}$ & $\mathscr{T}^{3}$ & $\mathscr{T}^{4}$ & $\mathscr{T}^{5}$ \\
\hline BS Hons. & $(0.4,0.5,0.8)$ & $(0.7,0.6,0.4)$ & $(0.4,0.5,0.7)$ & $(0.5,0.3,0.7)$ & $(0.5,0.3,0.8)$ \\
\hline MS/MPhil. & $(0.5,0.3,0.6)$ & $(0.3,0.2,0.1)$ & $(0.3,0.6,0.2)$ & $(0.7,0.3,0.6)$ & $(0.5,0.4,0.5)$ \\
\hline Ph.D. & $(0.8,0.2,0.4)$ & $(0.9,0.5,0.3)$ & $(0.9,0.4,0.1)$ & $(0.6,0.3,0.2)$ & $(0.6,0.1,0.2)$ \\
\hline Postdoctorate & $(0.9,0.3,0.1)$ & $(0.5,0.2,0.1)$ & $(0.8,0.5,0.2)$ & $(0.8,0.2,0.1)$ & $(0.7,0.4,0.2)$ \\
\hline
\end{tabular}

TABLE 3: Opinion of decision-makers for all alternatives against $\mathscr{A}_{2}^{b}$.

\begin{tabular}{|c|c|c|c|c|c|}
\hline $\mathscr{A}_{2}^{b}$ (Experience $\left.(\mathbf{y r})\right)$ & $\mathscr{T}^{1}$ & $\mathscr{T}^{2}$ & $\mathscr{T}^{3}$ & $\mathscr{T}^{4}$ & $\mathscr{T}^{5}$ \\
\hline 5 & $(0.3,0.4,0.7)$ & $(0.6,0.5,0.3)$ & $(0.5,0.6,0.8)$ & $(0.6,0.4,0.8)$ & $(0.3,0.6,0.7)$ \\
\hline 8 & $(0.4,0.2,0.5)$ & $(0.8,0.1,0.2)$ & $(0.4,0.7,0.3)$ & $(0.4,0.8,0.7)$ & $(0.7,0.5,0.6)$ \\
\hline 10 & $(0.7,0.2,0.3)$ & $(0.9,0.3,0.1)$ & $(0.8,0.3,0.2)$ & $(0.5,0.4,0.3)$ & $(0.5,0.2,0.1)$ \\
\hline 15 & $(0.8,0.2,0.1)$ & $(0.6,0.4,0.3)$ & $(0.9,0.4,0.1)$ & $(0.6,0.2,0.3)$ & $(0.5,0.3,0.2)$ \\
\hline
\end{tabular}

TABLE 4: Opinion of decision-makers for all alternatives against $\mathscr{A}_{3}^{c}$.

\begin{tabular}{lcccrr}
\hline $\mathscr{A}_{3}^{c}$ (Gender) & $\mathscr{T}^{1}$ & $\mathscr{T}^{2}$ & $\mathscr{T}^{3}$ & $\mathscr{T}^{4}$ & $\mathscr{T}^{5}$ \\
\hline Male & $(0.5,0.6,0.9)$ & $(0.7,0.8,0.3)$ & $(0.6,0.4,0.3)$ & $(0.8,0.5,0.4)$ & $(0.9,0.2,0.1)$ \\
Female & $(0.6,0.4,0.7)$ & $(0.3,0.6,0.4)$ & $(0.8,0.2,0.1)$ & $(0.4,0.5,0.6)$ & $(0.8,0.4,0.2)$ \\
\hline
\end{tabular}

TABLE 5: Opinion of decision-makers for all alternatives against $\mathscr{A}_{4}^{d}$.

\begin{tabular}{lcccrr}
\hline $\mathscr{A}_{4}^{d}$ (Publication) & $\mathscr{T}^{1}$ & $\mathscr{T}^{2}$ & $\mathscr{T}^{3}$ & $\mathscr{T}^{4}$ & $\mathscr{T}^{5}$ \\
\hline 3 & $(0.6,0.4,0.5)$ & $(0.7,0.5,0.3)$ & $(0.6,0.4,0.3)$ & $(0.6,0.2,0.1)$ & $(0.4,0.5,0.3)$ \\
5 & $(0.8,0.2,0.4)$ & $(0.7,0.3,0.2)$ & $(0.8,0.3,0.1)$ & $(0.3,0.4,0.5)$ & $(0.3,0.5,0.8)$ \\
8 & $(0.5,0.3,0.4)$ & $(0.6,0.3,0.4)$ & $(0.5,0.7,0.2)$ & $(0.8,0.4,0.1)$ & $(0.7,0.4,0.3)$ \\
$10+$ & $(0.4,0.9,0.6)$ & $(0.8,0.4,0.2)$ & $(0.2,0.6,0.5)$ & $(0.7,0.5,0.2)$ & $(0.6,0.4,0.7)$ \\
\hline
\end{tabular}

TABLE 6: Characteristic function of NHSS.

\begin{tabular}{lcccr}
\hline & $\mathscr{A}_{1}^{a}$ & $\mathscr{A}_{2}^{b}$ & $\mathscr{A}_{3}^{c}$ & $\mathscr{A}_{4}^{d}$ \\
\hline $\mathscr{T}^{1}$ & $(0.5,0.3,0.6)$ & $(0.3,0.4,0.7)$ & $(0.5,0.6,0.9)$ & $(0.6,0.4,0.5)$ \\
$\mathscr{T}^{2}$ & $(0.3,0.2,0.1)$ & $(0.6,0.5,0.3)$ & $(0.7,0.8,0.3)$ & $(0.7,0.5,0.3)$ \\
$\mathscr{T}^{4}$ & $(0.7,0.3,0.6)$ & $(0.6,0.4,0.8)$ & $(0.8,0.5,0.4)$ & $(0.6,0.2,0.1)$ \\
$\mathscr{T}^{5}$ & $(0.5,0.4,0.5)$ & $(0.3,0.6,0.7)$ & $(0.9,0.2,0.1)$ & $(0.4,0.5,0.3)$ \\
\hline
\end{tabular}

and $\mathbf{0 . 1}$ be the scalar, then scalar multiplication of NHSM

$[O]_{4 \times 4}$ is given as

$$
[(0.1) O]_{4 \times 4}=\left[\begin{array}{ccccc}
(0.05,0.03,0.06) & (0.03,0.04,0.07) & (0.03,0.04,0.07) & (0.06,0.04,0.05) \\
(0.03,0.02,0.01) & (0.06,0.05,0.03) & (0.07,0.08,0.03) & (0.07,0.05,0.03) \\
(0.07,0.03,0.06) & (0.06,0.04,0.08) & (0.08,0.05,0.04) & (0.06,0.02,0.01) \\
(0.05,0.04,0.05) & (0.03,0.06,0.07) & (0.09,0.02,0.01) & (0.04,0.05,0.03)
\end{array}\right]
$$

Proposition 1. Let $O=\left[O_{i j}\right]$ and $\mathscr{M}=\left[\mathscr{M}_{i j}\right]$ be two NHSMs, where $O_{i j}=\left(\mathscr{T}_{i j k}^{o}, \mathscr{J}_{i j k}^{o}, \mathscr{F}_{i j k}^{o}\right)$ and $\mathscr{M}_{i j}=\left(\mathscr{T}_{i j k}^{M}\right.$, $\left.\mathscr{I}_{i j k}^{\mathscr{M}}, \mathscr{F}_{i j k}^{M}\right)$. For two scalars $s, t \in[0,1]$, then
(1) $s(t O)=(s t) O$
(2) If $s<t$, then $s O<t O$.
(3) If $O \subseteq \mathscr{M}$, then $s O \subseteq s \mathscr{M}$.

$$
\begin{aligned}
& s(t O)=s\left[t O_{i j}\right]=s\left[\left(t \mathscr{T}_{i j k}^{o}, t \mathscr{F}_{i j k}^{o}, t \mathscr{F}_{i j k}^{o}\right)\right] \\
& =\left[\left(s t \mathscr{T}_{i j k}^{o}, s t \mathscr{F}_{i j k}^{o}, s t \mathscr{F}_{i j k}^{o}\right)\right]=s t\left[\left(\mathscr{T}_{i j k}^{o}, \mathscr{I}_{i j k}^{o}, \mathscr{F}_{i j k}^{o}\right)\right] \\
& =s t\left[O_{i j}\right]=(s t) O .
\end{aligned}
$$

Since $\mathscr{T}_{i j k}^{o}, \mathscr{J}_{i j k}^{o}, \mathscr{F}_{i j k}^{o} \in[0,1]$, then $s \mathscr{T}_{i j k}^{o} \leq t \mathscr{T}_{i j k}^{o}$, $s \mathscr{J}_{i j k}^{o} \leq t \mathscr{J}_{i j k}^{o}, s \mathscr{F}_{i j k}^{o} \leq t \mathscr{F}_{i j k}^{o}$.

Now, 


$$
\begin{aligned}
s O & =\left[s O_{i j}\right]=\left[\left(s \mathscr{T}_{i j k}^{o}, s \mathscr{F}_{i j k}^{o}, s \mathscr{F}_{i j k}^{o}\right)\right] \leq\left[\left(t \mathscr{T}_{i j k}^{o}, t \mathscr{F}_{i j k}^{o}, t \mathscr{F}_{i j k}^{o}\right)\right]=\left[t O_{i j}\right]=t O, \\
O \subseteq \mathscr{M} \Rightarrow\left[O_{i j}\right] \subseteq\left[\mathscr{M}_{i j}\right] & \\
& \Rightarrow \mathscr{T}_{i j k}^{o} \leq \mathscr{T}_{i j k}^{\mathscr{M}}, \mathscr{F}_{i j k}^{o} \leq \mathscr{I}_{i j k}^{\mathscr{M}}, \mathscr{F}_{i j k}^{o} \geq \mathscr{F}_{i j k}^{\mathscr{M}} \\
& \Rightarrow s \mathscr{T}_{i j k}^{o} \leq s \mathscr{T}_{i j k}^{\mathscr{M}}, s \mathscr{F}_{i j k}^{o} \leq s \mathscr{J}_{i j k}^{\mathscr{M}}, s \mathscr{F}_{i j k}^{o} \geq s \mathscr{F}_{i j k}^{\mathscr{M}} \\
& \Rightarrow s\left[O_{i j}\right] \subseteq s\left[\mathscr{M}_{i j}\right] \\
& \Rightarrow s O \subseteq s \mathscr{M} .
\end{aligned}
$$

Similarly, we can prove assertions 1 and 2 .

Theorem 1. Let $O=\left[O_{i j}\right]$ be the NHSM of order $\alpha \times \beta$, where $O_{i j}=\left(\mathscr{T}_{i j k}^{o}, \mathscr{I}_{i j k}^{o}, \mathscr{F}_{i j k}^{o}\right)$. Then,

(1) $(s O)^{t}=s O^{t}$, where $s \in[0,1]$.

(2) $\left(O^{t}\right)^{t}=O$.

(3) If $O=\left[O_{i j}\right]$ is the upper triangular NHSM, then $O^{t}$ is lower triangular NHSM and vice versa.

Proof. Here, $(s O)^{t}, s \mathrm{O}^{t} \in \mathrm{NHSM}_{\alpha \times \beta}$, so

$$
\begin{aligned}
(s O)^{t} & =\left[\left(s \mathscr{T}_{i j k}^{o}, s \mathscr{J}_{i j k}^{o}, s \mathscr{F}_{i j k}^{o}\right)\right]^{t} \\
& =\left[\left(s \mathscr{T}_{j k i}^{o}, s \mathscr{J}_{j k i}^{o}, s \mathscr{F}_{j k i}^{o}\right)\right] \\
& =s\left[\left(\mathscr{T}_{j k i}^{o}, \mathscr{J}_{j k i}^{o}, \mathscr{F}_{j k i}^{o}\right)\right] \\
& =s\left[\left(\mathscr{T}_{i j k}^{o}, \mathscr{J}_{i j k}^{o}, \mathscr{F}_{i j k}^{o}\right)\right]^{t}=s O^{t} .
\end{aligned}
$$

Proof. Since $O^{t} \in \mathrm{NHSM}_{\alpha \times \beta}$, then $\left(O^{t}\right)^{t} \in \mathrm{NHSM}_{\alpha \times \beta}$. Now,

$$
\begin{aligned}
\left(O^{t}\right)^{t} & =\left(\left[\left(\mathscr{T}_{i j k}^{o}, \mathscr{J}_{i j k}^{o}, \mathscr{F}_{i j k}^{o}\right)\right]^{t}\right)^{t} \\
& =\left(\left[\left(\mathscr{T}_{j k i}^{o}, \mathscr{J}_{j k i}^{o}, \mathscr{F}_{j k i}^{o}\right)\right]\right)^{t} \\
& =\left[\left(\mathscr{T}_{i j k}^{o}, \mathscr{F}_{i j k}^{o}, \mathscr{F}_{i j k}^{o}\right)\right]=O .
\end{aligned}
$$

Definition 11. Let $O=\left[O_{i j}\right]$ be the square NHSM of order $\alpha \times \beta$, where $O_{i j}=\left(\mathscr{T}_{i j k}^{o}, \mathscr{I}_{i j k}^{o}, \mathscr{F}_{i j k}^{o}\right)$ and $\alpha=\beta$. Then, the trace of NHSM is denoted as $\operatorname{tr}(O)$ and is defined as $\operatorname{tr}(O)=\sum_{i=1, k=a}^{\alpha, z}\left[\mathscr{T}_{i i k}^{o}-\left(\mathscr{I}_{i i k}^{o}+\mathscr{F}_{i i k}^{o}\right)\right]$.

Example 3. Let us consider an NHSM $[O]_{4 \times 4}$.

$$
[O]_{4 \times 4}=\left[\begin{array}{cccc}
(0.5,0.3,0.6) & (0.3,0.4,0.7) & (0.5,0.6,0.9) & (0.6,0.4,0.5) \\
(0.3,0.2,0.1) & (0.6,0.5,0.3) & (0.7,0.8,0.3) & (0.7,0.5,0.3) \\
(0.7,0.3,0.6) & (0.6,0.4,0.8) & (0.8,0.5,0.4) & (0.6,0.2,0.1) \\
(0.5,0.4,0.5) & (0.3,0.6,0.7) & (0.9,0.2,0.1) & (0.4,0.5,0.3)
\end{array}\right]
$$

Then, $\quad \operatorname{tr}(O)=(0.5-0.3-0.6)+(0.6-0.5-0.3)+\quad$ Proof $(0.8-0.5-0.4)+(0.4-0.5-0.3)=-1.1$.

Proposition 2. Let $O=\left[O_{i j}\right]$ be the square NHSM of order $\alpha \times \beta$, where $O_{i j}=\left(\mathscr{T}_{i j k}^{o}, \mathscr{I}_{i j k}^{o}, \mathscr{F}_{i j k}^{o}\right)$ and $\alpha=\beta$, and $s$ be any scalar, then $\operatorname{tr}(s O)=\operatorname{str}(O)$.

$$
\begin{aligned}
\operatorname{tr}(s O) & =\sum_{i=1, k=a}^{\alpha, z}\left[s \mathscr{T}_{i i k}^{o}-\left(s \mathscr{F}_{i i k}^{o}+s \mathscr{F}_{i i k}^{o}\right)\right] \\
& =s \sum_{i=1, k=a}^{\alpha, z}\left[\mathscr{T}_{i i k}^{o}-\left(\mathscr{F}_{i i k}^{o}+\mathscr{F}_{i i k}^{o}\right)\right] \\
& =s \operatorname{tr}(O) .
\end{aligned}
$$


Definition 12. Let $O=\left[O_{i j}\right]$ and $\mathscr{M}=\left[\mathscr{M}_{j m}\right]$ be two NHSMs, where $O_{i j}=\left(\mathscr{T}_{i j k}^{o}, \mathscr{J}_{i j k}^{o}, \mathscr{F}_{i j k}^{o}\right)$ and $\mathscr{M}_{j m}=\left(\mathscr{T}_{j k m}^{\mathscr{M}}\right.$, $\left.\mathscr{I}_{j k m}^{\mathscr{M}}, \mathscr{F}_{j k m}^{\mathscr{M}}\right)$. Then, $O$ and $\mathscr{M}$ are said to be conformable if their dimensions are equal to each other (the number of columns of $O$ is equal to several rows of $\mathscr{M})$. If $O=\left[O_{i j}\right]_{\alpha \times \beta}$ and $\mathscr{M}=\left[\mathscr{M}_{j m}\right]_{\beta \times \gamma}$, then $O \mathscr{M}=\left[\mathcal{S}_{i m}\right]_{\alpha \times \gamma}$, where

$$
\left[\mathcal{S}_{i m}\right]=\left(\max _{j k} \min \left(\mathscr{T}_{i j k}^{o}, \mathscr{T}_{j k m}^{\mathscr{M}}\right), \min _{j k} \max \left(\mathscr{J}_{i j k}^{o}, \mathscr{I}_{j k m}^{\mathscr{M}}\right), \min _{j k} \max \left(\mathscr{F}_{i j k}^{o}, \mathscr{F}_{j k m}^{\mathscr{M}}\right)\right) .
$$

Theorem 2. Let $O=\left[O_{i j}\right]_{\alpha \times \beta}$ and $\mathscr{M}=\left[\mathscr{M}_{j m}\right]_{\beta \times \gamma}$ be two NHSMs, where $O_{i j}=\left(\mathscr{T}_{i j k}^{o}, \mathscr{J}_{i j k}^{o}, \mathscr{F}_{i j k}^{o}\right)$ and $\mathscr{M}_{j m}=\left(\mathscr{T}_{j k m}^{\mathscr{M}}\right.$, $\left.\mathscr{I}_{j k m}^{\mathscr{M}}, \mathscr{F}_{j k m}^{\mathscr{M}}\right)$. Then, $(\mathrm{O} \boldsymbol{M})^{t}=\mathscr{M}^{t} \mathbf{\square} \mathrm{O}^{t}$.
Proof. Let $O \mathbf{\square} \mathscr{M}=\left[\mathcal{S}_{i m}\right]_{\alpha \times \gamma}$, then $(O \mathbf{M} \mathscr{M})^{t}=\left[\mathcal{S}_{m i}\right]_{\gamma \times \alpha}, O^{t}$ $=\left[O_{j i}\right]_{\beta \times \alpha}, \mathscr{M}^{t}=\left[\mathscr{M}_{m j}\right]_{\gamma \times \beta}$.

Now,

$$
\begin{aligned}
(O \mathbf{M})^{t} & =\left(\mathscr{T}_{k m i}^{\mathcal{S}}, \mathscr{J}_{k m i}^{\mathcal{S}}, \mathscr{F}_{k m i}^{\mathcal{S}}\right)_{\gamma \times \alpha} \\
& =\left(\max _{j k} \min \left(\mathscr{T}_{m j k}^{\mathscr{M}}, \mathscr{T}_{j k i}^{o}\right), \min _{j k} \max \left(\mathscr{I}_{m j k}^{\mathscr{M}}, \mathscr{J}_{j k i}^{o}\right), \min _{j k} \max \left(\mathscr{F}_{m j k}^{\mathscr{M}}, \mathscr{F}_{j k i}^{0}\right)\right)_{\gamma \times \alpha} \\
& =\left(\mathscr{T}_{m j k}^{\mathscr{M}}, \mathscr{I}_{m j k}^{\mathscr{M}}, \mathscr{F}_{m j k}^{\mathscr{M}}\right)_{\gamma \times \beta} \boldsymbol{\square}\left(\mathscr{T}_{j k i}^{o}, \mathscr{J}_{j k i}^{o}, \mathscr{F}_{j k i}^{0}\right)_{\beta \times \alpha} \\
& =\mathscr{M}^{t} \mathbf{\square} O^{t} .
\end{aligned}
$$

Definition 13. Let $O=\left[O_{i j}\right]$ and $\mathscr{M}=\left[\mathscr{M}_{i j}\right]$ be two NHSMs, where $\quad O_{i j}=\left(\mathscr{T}_{i j k}^{o}, \mathscr{J}_{i j k}^{o}, \mathscr{F}_{i j k}^{o}\right) \quad$ and

$$
O \cup \mathscr{M}=\mathcal{S}, \quad \text { where } \mathscr{T}_{i j k}^{s}=\max \left(\mathscr{T}_{i j k}^{o}, \mathscr{T}_{i j k}^{\mathscr{M}}\right), \mathscr{I}_{i j k}^{s}=\frac{\left(\mathscr{I}_{i j k}^{o}+\mathscr{T}_{i j k}^{\mathscr{M}}\right)}{2}, \mathscr{F}_{i j k}^{s}=\min \left(\mathscr{F}_{i j k}^{o}, \mathscr{F}_{i j k}^{\mathscr{M}}\right) .
$$

Definition 14. Let $O=\left[O_{i j}\right]$ and $\mathscr{M}=\left[\mathscr{M}_{i j}\right]$ be two NHSMs, where $O_{i j}=\left(\mathscr{T}_{i j k}^{o}, \mathscr{I}_{i j k}^{o}, \mathscr{F}_{i j k}^{o}\right)$ and $\mathscr{M}_{i j}=\left(\mathscr{T}_{i j k}^{\mathscr{M}}\right.$, $\left.\mathscr{I}_{i j k}^{\mathscr{M}}, \mathscr{F}_{i j k}^{\mathscr{M}}\right)$. Then, their intersection is defined as follows:

$$
O \cap \mathscr{M}=\mathcal{S}, \quad \text { where } \mathscr{T}_{i j k}^{s}=\min \left(\mathscr{T}_{i j k}^{o}, \mathscr{T}_{i j k}^{\mathscr{M}}\right), \mathscr{J}_{i j k}^{s}=\frac{\left(\mathscr{F}_{i j k}^{o}+\mathscr{I}_{i j k}^{\mathscr{M}}\right)}{2}, \mathscr{F}_{i j k}^{s}=\max \left(\mathscr{F}_{i j k}^{o}, \mathscr{F}_{i j k}^{\mathscr{M}}\right) .
$$

Proposition 3. Let $O=\left[O_{i j}\right]$ and $\mathscr{M}=\left[\mathscr{M}_{i j}\right]$ be two upper triangular NHSMs, where $O_{i j}=\left(\mathscr{T}_{i j k}^{o}, \mathscr{J}_{i j k}^{o}, \mathscr{F}_{i j k}^{o}\right)$ and

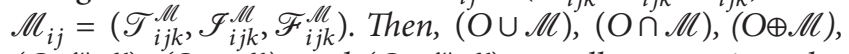
$\left(O \oplus^{w} \mathscr{M}\right),(\mathrm{\odot} \odot \mathscr{M})$, and $\left(O \odot{ }^{w} \mathscr{M}\right)$ are all upper triangular NHSMs and vice versa.
Theorem 3. Let $O=\left[O_{i j}\right]$ and $\mathscr{M}=\left[\mathscr{M}_{i j}\right]$ be two NHSMs, where $O_{i j}=\left(\mathscr{T}_{i j k}^{o}, \mathscr{J}_{i j k}^{o}, \mathscr{F}_{i j k}^{o}\right)$ and $\mathscr{M}_{i j}=\left(\mathscr{T}_{i j k}^{\mathscr{M}}, \mathscr{J}_{i j k}^{\mathscr{M}}, \mathscr{F}_{i j k}^{\mathscr{M}}\right)$. Then,

(1) $(O \cup \mathscr{M})^{\diamond}=O^{\diamond} \cap \mathscr{M}^{\diamond}$. 
(2) $(O \cap \mathscr{M})^{\diamond}=O^{\diamond} \cup \mathscr{M}^{\diamond}$.

Proof

$$
\begin{aligned}
(O \cup \mathscr{M})^{\diamond} & =\left[\left(\max \left(\mathscr{T}_{i j k}^{o}, \mathscr{T}_{i j k}^{\mathscr{M}}\right), \frac{\left(\mathscr{F}_{i j k}^{o}+\mathscr{I}_{i j k}^{\mathscr{M}}\right)}{2}, \min \left(\mathscr{F}_{i j k}^{o}, \mathscr{F}_{i j k}^{\mathscr{M}}\right)\right)\right]^{\diamond} \\
& =\left[\left(\min \left(\mathscr{F}_{i j k}^{o}, \mathscr{F}_{i j k}^{\mathscr{M}}\right), \frac{\left(\mathscr{F}_{i j k}^{o}+\mathscr{F}_{i j k}^{\mathscr{M}}\right)}{2}, \max \left(\mathscr{T}_{i j k}^{o}, \mathscr{T}_{i j k}^{\mathscr{M}}\right)\right)\right] \\
& =\left(\mathscr{F}_{i j k}^{o}, \mathscr{J}_{i j k}^{o}, \mathscr{T}_{i j k}^{o}\right) \cap\left(\mathscr{F}_{i j k}^{\mathscr{M}}, \mathscr{F}_{i j k}^{\mathscr{M}}, \mathscr{T}_{i j k}^{\mathscr{M}}\right) \\
& =\left(\mathscr{T}_{i j k}^{o}, \mathscr{F}_{i j k}^{o}, \mathscr{F}_{i j k}^{o}\right)^{\diamond} \cap\left(\mathscr{T}_{i j k}^{\mathscr{M}}, \mathscr{F}_{i j k}^{\mathscr{M}}, \mathscr{F}_{i j k}^{\mathscr{M}}\right)^{\diamond} \\
& =O^{\diamond} \cap \mathscr{M}^{\diamond} .
\end{aligned}
$$

Proof

$$
\begin{aligned}
(O \cap \mathscr{M})^{\diamond} & =\left[\left(\min \left(\mathscr{T}_{i j k}^{o}, \mathscr{T}_{i j k}^{\mathscr{M}}\right), \frac{\left(\mathscr{F}_{i j k}^{o}+\mathscr{F}_{i j k}^{\mathscr{M}}\right)}{2}, \max \left(\mathscr{F}_{i j k}^{o}, \mathscr{F}_{i j k}^{\mathscr{M}}\right)\right)\right]^{\diamond} \\
& =\left[\left(\max \left(\mathscr{F}_{i j k}^{o}, \mathscr{F}_{i j k}^{\mathscr{M}}\right), \frac{\left(\mathscr{F}_{i j k}^{o}+\mathscr{F}_{i j k}^{\mathscr{M}}\right)}{2}, \min \left(\mathscr{T}_{i j k}^{o}, \mathscr{T}_{i j k}^{\mathscr{M}}\right)\right)\right] \\
& =\left(\mathscr{F}_{i j k}^{o}, \mathcal{F}_{i j k}^{o}, \mathscr{T}_{i j k}^{o}\right) \cup\left(\mathscr{F}_{i j k}^{\mathscr{M}}, \mathscr{F}_{i j k}^{\mathscr{M}}, \mathscr{T}_{i j k}^{\mathscr{M}}\right) \\
& =\left(\mathscr{T}_{i j k}^{o}, \mathscr{J}_{i j k}^{o}, \mathscr{F}_{i j k}^{o}\right)^{\diamond} \cup\left(\mathscr{T}_{i j k}^{\mathscr{M}}, \mathscr{F}_{i j k}^{\mathscr{M}}, \mathscr{F}_{i j k}^{\mathscr{M}}\right)^{\diamond} \\
& =O^{\diamond} \cup \mathscr{M}^{\diamond} .
\end{aligned}
$$

Theorem 4. Let $O=\left[O_{i j}\right]$ and $\mathscr{M}=\left[\mathscr{M}_{i j}\right]$ be two NHSMs, where $O_{i j}=\left(\mathscr{T}_{i j k}^{o}, \mathscr{J}_{i j k}^{o}, \mathscr{F}_{i j k}^{o}\right)$ and $\mathscr{M}_{i j}=\left(\mathscr{T}_{i j k}^{\mathscr{M}}, \mathscr{J}_{i j k}^{\mathscr{M}}, \mathscr{F}_{i j k}^{\mathscr{M}}\right)$. Then,

(1) $(O \cup \mathscr{M})=(\mathscr{M} \cup O)$.

(2) $(O \cap \mathscr{M})=(\mathscr{M} \cap O)$.

$$
\begin{aligned}
(O \cup \mathscr{M}) & =\left[\left(\max \left(\mathscr{T}_{i j k}^{o}, \mathscr{T}_{i j k}^{\mathscr{M}}\right), \frac{\left(\mathscr{F}_{i j k}^{o}+\mathscr{F}_{i j k}^{\mathscr{M}}\right)}{2}, \min \left(\mathscr{F}_{i j k}^{o}, \mathscr{F}_{i j k}^{\mathscr{M}}\right)\right)\right] \\
& =\left[\left(\max \left(\mathscr{T}_{i j k}^{\mathscr{M}}, \mathscr{T}_{i j k}^{o}\right), \frac{\left(\mathscr{J}_{i j k}^{\mathscr{M}}+\mathscr{F}_{i j k}^{o}\right)}{2}, \min \left(\mathscr{F}_{i j k}^{\mathscr{M}}, \mathscr{F}_{i j k}^{o}\right)\right)\right] \\
& =\left(\mathscr{T}_{i j k}^{\mathscr{M}}, \mathscr{F}_{i j k}^{\mathscr{M}}, \mathscr{F}_{i j k}^{\mathscr{M}}\right) \cup\left(\mathscr{T}_{i j k}^{o}, \mathscr{F}_{i j k}^{o}, \mathscr{F}_{i j k}^{o}\right) \\
& =(\mathscr{M} \cup O) .
\end{aligned}
$$




$$
\begin{aligned}
(O \cap \mathscr{M}) & =\left[\left(\min \left(\mathscr{T}_{i j k}^{o}, \mathscr{T}_{i j k}^{\mathscr{M}}\right), \frac{\left(\mathscr{F}_{i j k}^{o}+\mathscr{I}_{i j k}^{\mathscr{M}}\right)}{2}, \max \left(\mathscr{F}_{i j k}^{o}, \mathscr{F}_{i j k}^{\mathscr{M}}\right)\right)\right] \\
& =\left[\left(\min \left(\mathscr{T}_{i j k}^{\mathscr{M}}, \mathscr{T}_{i j k}^{o}\right), \frac{\left(\mathscr{F}_{i j k}^{\mathscr{M}}+\mathscr{F}_{i j k}^{o}\right)}{2}, \max \left(\mathscr{F}_{i j k}^{\mathscr{M}}, \mathscr{F}_{i j k}^{o}\right)\right)\right] \\
& =\left(\mathscr{T}_{i j k}^{\mathscr{M}}, \mathscr{I}_{i j k}^{\mathscr{M}}, \mathscr{F}_{i j k}^{\mathscr{M}}\right) \cap\left(\mathscr{T}_{i j k}^{o}, \mathscr{F}_{i j k}^{o}, \mathscr{F}_{i j k}^{o}\right) \\
& =(\mathscr{M} \cap O) .
\end{aligned}
$$

Theorem 5. Let $O=\left[O_{i j}\right], \mathscr{M}=\left[\mathscr{M}_{i j}\right]$, and $\mathcal{N}=\left[\mathscr{M}_{i j}\right]$ be

NHSM, where $O_{i j}=\left(\mathscr{T}_{i j k}^{o}, \mathscr{J}_{i j k}^{o}, \mathscr{F}_{i j k}^{o}\right), \mathscr{M}_{i j}=\left(\mathscr{T}_{i j k}^{\mathscr{M}}, \mathscr{I}_{i j k}^{\mathscr{M}}\right.$, $\left.\mathscr{F}_{i j k}^{\mathscr{M}}\right)$, and $\mathscr{N}_{i j}=\left(\mathscr{T}_{i j k}^{\mathcal{N}}, \mathscr{J}_{i j k}^{\mathcal{N}}, \mathscr{F}_{i j k}^{\mathcal{N}}\right)$. Then,

$$
\begin{aligned}
& (O \cup \mathscr{M}) \cup \mathcal{N}=O \cup(\mathscr{M} \cup \mathcal{N}), \\
& (O \cap \mathscr{M}) \cap \mathcal{N}=O \cap(\mathscr{M} \cap \mathcal{N}) .
\end{aligned}
$$

$$
\begin{aligned}
& (O \cup \mathscr{M}) \cup \mathcal{N}=\left[\left(\max \left(\mathscr{T}_{i j k}^{o}, \mathscr{T}_{i j k}^{\mathscr{M}}\right), \frac{\left(\mathscr{F}_{i j k}^{o}+\mathscr{I}_{i j k}^{\mathscr{M}}\right)}{2}, \min \left(\mathscr{F}_{i j k}^{o}, \mathscr{F}_{i j k}^{\mathscr{M}}\right)\right)\right] \cup\left[\left(\mathscr{T}_{i j k}^{\mathcal{N}}, \mathscr{J}_{i j k}^{\mathcal{N}}, \mathscr{F}_{i j k}^{\mathcal{N}}\right)\right] \\
& =\left[\left(\max \left(\mathscr{T}_{i j k}^{o}, \mathscr{T}_{i j k}^{\mathscr{M}}, \mathscr{T}_{i j k}^{\mathcal{N}}\right), \frac{\left(\mathscr{J}_{i j k}^{o}+\mathscr{I}_{i j k}^{\mathscr{M}}+\mathscr{I}_{i j k}^{\mathcal{N}}\right)}{3}, \min \left(\mathscr{F}_{i j k}^{o}, \mathscr{F}_{i j k}^{\mathscr{M}}, \mathscr{F}_{i j k}^{\mathcal{N}}\right)\right)\right] \\
& =\left[\left(\max \left(\mathscr{T}_{i j k}^{O}, \mathscr{T}_{i j k}^{M}, \mathscr{T}_{i j k}^{\mathcal{N}}\right), \frac{\left(\mathscr{I}_{i j k}^{O}+\mathscr{I}_{i j k}^{\mathscr{M}}+\mathscr{I}_{i j k}^{\mathcal{N}}\right)}{3}, \min \left(\mathscr{F}_{i j k}^{O}, \mathscr{F}_{i j k}^{\mathscr{M}}, \mathscr{F}_{i j k}^{\mathcal{N}}\right)\right)\right] \\
& =\left(\mathscr{T}_{i j k}^{O}, \mathscr{J}_{i j k}^{O}, \mathscr{F}_{i j k}^{O}\right) \cup\left[\left(\max \left(\mathscr{T}_{i j k}^{\mathscr{M}}, \mathscr{T}_{i j k}^{\mathcal{N}}\right), \frac{\left(\mathscr{I}_{i j k}^{\mathscr{M}}+\mathscr{I}_{i j k}^{\mathcal{N}}\right)}{2}, \min \left(\mathscr{F}_{i j k}^{\mathscr{M}}, \mathscr{F}_{i j k}^{\mathcal{N}}\right)\right)\right] \\
& =\left(\mathscr{T}_{i j k}^{O}, \mathscr{J}_{i j k}^{O}, \mathscr{F}_{i j k}^{O}\right) \cup\left(\left(\mathscr{T}_{i j k}^{\mathscr{M}}, \mathscr{J}_{i j k}^{\mathscr{M}}, \mathscr{F}_{i j k}^{\mathscr{M}}\right) \cup\left(\mathscr{T}_{i j k}^{\mathcal{N}}, \mathscr{J}_{i j k}^{\mathcal{N}}, \mathscr{F}_{i j k}^{\mathcal{N}}\right)\right) \\
& =O \cup(\mathscr{M} \cup \mathcal{N}) \text {. }
\end{aligned}
$$

Assertion 2 is similar to assertion 1 .

\section{Logical Operations for NHSMs with Their Properties}

In this section, we develop some logical operators for NHSM with their desirable properties and also introduce the notions of necessity and possibility operators for NHSM.

Definition 15. Let $O=\left[O_{i j}\right]$ and $\mathscr{M}=\left[\mathscr{M}_{i j}\right]$ be two NHSMs, where $O_{i j}=\left(\mathscr{T}_{i j k}^{o}, \mathscr{J}_{i j k}^{o}, \mathscr{F}_{i j k}^{o}\right)$ and $\mathscr{M}_{i j}=\left(\mathscr{T}_{i j k}^{\mathscr{M}}\right.$,
$\left.\mathscr{I}_{i j k}^{\mathscr{M}}, \mathscr{F}_{i j k}^{\mathscr{M}}\right)$. Then, OR-operation between them is defined as follows:

$$
\begin{aligned}
& \mathscr{T}(O \vee \mathscr{M})=\max \left\{\mathscr{T}_{i j k}^{O}, \mathscr{T}_{i j k}^{\mathscr{M}}\right\}, \\
& \mathscr{I}(O \vee \mathscr{M})=\min \left\{\mathscr{J}_{i j k}^{O}, \mathscr{J}_{i j k}^{\mathscr{M}}\right\}, \\
& \mathscr{F}(O \vee \mathscr{M})=\min \left\{\mathscr{F}_{i j k}^{O}, \mathscr{F}_{i j k}^{\mathscr{M}}\right\} .
\end{aligned}
$$

Definition 16. Let $O=\left[O_{i j}\right]$ and $\mathscr{M}=\left[\mathscr{M}_{i j}\right]$ be two NHSMs, where $\quad O_{i j}=\left(\mathscr{T}_{i j k}^{o}, \mathscr{J}_{i j k}^{o}, \mathscr{F}_{i j k}^{o}\right) \quad$ and $\mathscr{M}_{i j}=\left(\mathscr{T}_{i j k}^{\mathscr{M}}, \mathscr{I}_{i j k}^{\mathscr{M}}, \mathscr{F}_{i j k}^{\mathscr{M}}\right)$. Then, AND-operation between them is defined as follows: 


$$
\begin{aligned}
& \mathscr{T}(O \vee \mathscr{M})=\min \left\{\mathscr{T}_{i j k}^{O}, \mathscr{T}_{i j k}^{\mathscr{M}}\right\}, \\
& \mathscr{I}(O \vee \mathscr{M})=\max \left\{\mathscr{F}_{i j k}^{O}, \mathscr{F}_{i j k}^{\mathscr{M}}\right\}, \\
& \mathscr{F}(O \vee \mathscr{M})=\max \left\{\mathscr{F}_{i j k}^{O}, \mathscr{F}_{i j k}^{\mathscr{M}}\right\} .
\end{aligned}
$$

Proposition 4. Let $O=\left[O_{i j}\right], \mathscr{M}=\left[\mathscr{M}_{i j}\right]$, and $\mathscr{N}=\left[\mathscr{M}_{i j}\right]$ be three NHSMs, where $O_{i j}=\left(\mathscr{T}_{i j k}^{o}, \mathscr{F}_{i j k}^{o}, \mathscr{F}_{i j k}^{o}\right)$,

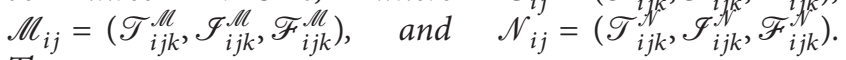
Then,

(1) $O \vee \mathscr{M}=\mathscr{M} \vee O$.

(2) $O \wedge \mathscr{M}=\mathscr{M} \wedge O$.

(3) $O \vee(\mathscr{M} \vee \mathcal{N})=(O \vee \mathscr{M}) \vee \mathcal{N}$

(4) $O \wedge(\mathscr{M} \wedge \mathcal{N})=(O \wedge \mathscr{M}) \wedge \mathcal{N}$.

Proof. The proof of the above proposition is straightforward.

Definition 17. Let $O=\left[O_{i j}\right]$ be an NHSM, where $O_{i j}=\left(\mathscr{T}_{i j k}^{o}, \mathscr{J}_{i j k}^{o}, \mathscr{F}_{i j k}^{o}\right)$. Then, the necessity operator for NHSM is represented as $\oplus O$ and defined as follows:

$$
\oplus O=\left(\mathscr{T}_{i j k}^{o}, \mathscr{J}_{i j k}^{o}, 1-\mathscr{T}_{i j k}^{o}\right) \text {. }
$$

Definition 18. Let $O=\left[O_{i j}\right]$ be an NHSM, where $O_{i j}=\left(\mathscr{T}_{i j k}^{o}, \mathscr{J}_{i j k}^{o}, \mathscr{F}_{i j k}^{o}\right)$. Then, the possibility operator for NHSM is represented as $\otimes O$ and defined as follows:

$$
\otimes O=\left(1-\mathscr{F}_{i j k}^{o}, \mathscr{J}_{i j k}^{o}, \mathscr{F}_{i j k}^{o}\right) .
$$

Proposition 5. Let $O=\left[O_{i j}\right]$ and $\mathscr{M}=\left[\mathscr{M}_{i j}\right]$ be two NHSMs, where $O_{i j}=\left(\mathscr{T}_{i j k}^{o}, \mathscr{F}_{i j k}^{o}, \mathscr{F}_{i j k}^{o}\right)$ and $\mathscr{M}_{i j}=\left(\mathscr{T}_{i j k}^{\mathscr{M}}, \mathscr{J}_{i j k}^{\mathscr{M}}, \mathscr{F}_{i j k}^{\mathscr{M}}\right)$. Then,
(1) $\oplus(O \cup \mathscr{M})=\oplus O \cup \oplus \mathscr{M}$
(2) $\oplus(O \cap \mathscr{M})=\oplus O \cap \oplus \mathscr{M}$.
(3) $\otimes(O \cup \mathscr{M})=\otimes O \cup \otimes \mathscr{M}$.
(4) $\otimes(O \cap \mathscr{M})=\otimes O \cap \otimes \mathscr{M}$.

Proof. We know that $O=\left[O_{i j}\right]=\left(\mathscr{T}_{i j k}^{o}, \mathscr{J}_{i j k}^{o}, \mathscr{F}_{i j k}^{o}\right)$ and $\mathscr{M}=\left[\mathscr{M}_{i j}\right]=\left(\mathscr{T}_{i j k}^{\mathscr{M}}, \mathscr{J}_{i j k}^{\mathscr{M}}, \mathscr{F}_{i j k}^{\mathscr{M}}\right)$ are two NHSMs. Then, utilizing Definition 13, we get

$$
\begin{aligned}
& O \cup \mathscr{M}=\left(\mathscr{T}_{i j k}^{o}, \mathscr{F}_{i j k}^{o}, \mathscr{F}_{i j k}^{o}\right) \cup\left(\mathscr{T}_{i j k}^{\mathscr{M}}, \mathscr{F}_{i j k}^{\mathscr{M}}, \mathscr{F}_{i j k}^{\mathscr{M}}\right), \\
& O \cup \mathscr{M}=\left(\max \left(\mathscr{T}_{i j k}^{o}, \mathscr{T}_{i j k}^{\mathscr{M}}\right), \frac{\left(\mathscr{F}_{i j k}^{o}+\mathscr{F}_{i j k}^{\mathscr{M}}\right)}{2}, \min \left(\mathscr{F}_{i j k}^{o}, \mathscr{F}_{i j k}^{\mathscr{M}}\right)\right) .
\end{aligned}
$$

Utilizing Definition 17, we get

$$
\oplus(O \cup \mathscr{M})=\left(\max \left(\mathscr{T}_{i j k}^{o}, \mathscr{T}_{i j k}^{\mathscr{M}}\right), \frac{\left(\mathscr{F}_{i j k}^{o}+\mathscr{F}_{i j k}^{\mathscr{M}}\right)}{2}, 1-\max \left(\mathscr{T}_{i j k}^{o}, \mathscr{T}_{i j k}^{\mathscr{M}}\right)\right)
$$

Again, using Definition 17, we have

Then, utilizing Definition 13, we have

$$
\begin{aligned}
\oplus O & =\left(\mathscr{T}_{i j k}^{o}, \mathscr{J}_{i j k}^{o}, 1-\mathscr{T}_{i j k}^{o}\right), \\
\oplus \mathscr{M} & =\left(\mathscr{T}_{i j k}^{\mathscr{M}}, \mathscr{J}_{i j k}^{\mathscr{M}}, 1-\mathscr{T}_{i j k}^{\mathscr{M}}\right) .
\end{aligned}
$$

Hence,

$$
\oplus(O \cup \mathscr{M})=\oplus O \cup \oplus \mathscr{M}
$$

$$
\begin{aligned}
& \oplus O \cup \oplus \mathscr{M}=\left(\max \left(\mathscr{T}_{i j k}^{o}, \mathscr{T}_{i j k}^{\mathscr{M}}\right), \frac{\left(\mathcal{F}_{i j k}^{o}+\mathscr{F}_{i j k}^{\mathscr{M}}\right)}{2}, \min \left(1-\mathscr{T}_{i j k}^{o}, 1-\mathscr{T}_{i j k}^{M}\right)\right), \\
& \oplus O \cup \oplus \mathscr{M}=\left(\max \left(\mathscr{T}_{i j k}^{o}, \mathscr{T}_{i j k}^{\mathscr{M}}\right), \frac{\left(\mathcal{F}_{i j k}^{o}+\mathscr{F}_{i j k}^{\mathscr{M}}\right)}{2}, 1-\max \left(\mathscr{T}_{i j k}^{o}, \mathscr{T}_{i j k}^{\mathscr{M}}\right)\right) . \\
& \quad O \cup \mathscr{M}=\left(\mathscr{T}_{i j k}^{o}, \mathscr{F}_{i j k}^{o}, \mathscr{F}_{i j k}^{o}\right) \cup\left(\mathscr{T}_{i j k}^{\mathscr{M}}, \mathscr{F}_{i j k}^{\mathscr{M}}, \mathscr{F}_{i j k}^{\mathscr{M}}\right),
\end{aligned}
$$

Proof. It is similar to assertion 1.

Proof. We know that $O=\left[O_{i j}\right]=\left(\mathscr{T}_{i j k}^{o}, \mathscr{F}_{i j k}^{o}, \mathscr{F}_{i j k}^{o}\right)$ and $\mathscr{M}=\left[\mathscr{M}_{i j}\right]=\left(\mathscr{T}_{i j k}^{\mathscr{M}}, \mathscr{J}_{i j k}^{\mathscr{M}}, \mathscr{F}_{i j k}^{\mathscr{M}}\right)$ are two NHSMs. Then, utilizing Definition 14, we get

$$
O \cup \mathscr{M}=\left(\max \left(\mathscr{T}_{i j k}^{o}, \mathscr{T}_{i j k}^{\mathscr{M}}\right), \frac{\left(\mathscr{F}_{i j k}^{o}+\mathscr{F}_{i j k}^{M}\right)}{2}, \min \left(\mathscr{F}_{i j k}^{o}, \mathscr{F}_{i j k}^{\mathscr{M}}\right)\right) \text {. }
$$




$$
\otimes(O \cup \mathscr{M})=\left(1-\min \left(\mathscr{F}_{i j k}^{o}, \mathscr{F}_{i j k}^{\mathscr{M}}\right), \frac{\left(\mathscr{J}_{i j k}^{o}+\mathscr{I}_{i j k}^{\mathscr{M}}\right)}{2}, \min \left(\mathscr{F}_{i j k}^{o}, \mathscr{F}_{i j k}^{\mathscr{M}}\right)\right)
$$

Again, using Definition 18, we have

Then, utilizing Definition 13, we get

$$
\begin{aligned}
& \otimes O=\left(1-\mathscr{F}_{i j k}^{o}, \mathscr{F}_{i j k}^{o}, \mathscr{F}_{i j k}^{o}\right), \\
& \otimes \mathscr{M}=\left(1-\mathscr{F}_{i j k}^{\mathscr{M}}, \mathscr{F}_{i j k}^{\mathscr{M}}, \mathscr{F}_{i j k}^{\mathscr{M}}\right) .
\end{aligned}
$$

$$
\begin{aligned}
& \otimes O \cup \otimes \mathscr{M}=\left(\max \left(1-\mathscr{F}_{i j k}^{o}, 1-\mathscr{F}_{i j k}^{\mathscr{M}}\right), \frac{\left(\mathscr{J}_{i j k}^{o}+\mathscr{J}_{i j k}^{\mathscr{M}}\right)}{2}, \min \left(\mathscr{F}_{i j k}^{o}, \mathscr{F}_{i j k}^{\mathscr{M}}\right)\right), \\
& \otimes O \cup \otimes \mathscr{M}=\left(1-\min \left(\mathscr{F}_{i j k}^{o}, \mathscr{F}_{i j k}^{\mathscr{M}}\right), \frac{\left(\mathscr{F}_{i j k}^{o}+\mathscr{I}_{i j k}^{\mathscr{M}}\right)}{2}, \min \left(\mathscr{F}_{i j k}^{o}, \mathscr{F}_{i j k}^{\mathscr{M}}\right)\right) .
\end{aligned}
$$

Hence,

$$
\otimes(O \cup \mathscr{M})=\otimes O \cup \otimes \mathscr{M}
$$

Proof. It is similar to assertion 3.

Definition 19. Let $O=\left[O_{i j}\right]$ be the NHSM of order $\alpha \times \beta$, where $O_{i j}=\left(\mathscr{T}_{i j k}^{o}, \mathscr{J}_{i j k}^{o}, \mathscr{F}_{i j k}^{o}\right)$, then the value of matrix $O$ is denoted as $\mathscr{V}(O)$ and it is defined as $\mathscr{V}(O)=\left[\mathscr{V}_{i j}^{O}\right]$ of order $\alpha \times \beta$, where $\mathscr{V}_{i j}^{O}=\mathscr{T}_{i j k}^{o}-\mathscr{I}_{i j k}^{o},-\mathscr{F}_{i j k}^{o}$. The score of two NHSMs $O=\left[O_{i j}\right]$ and $\mathscr{M}=\left[\mathscr{M}_{i j}\right]$ of order $\alpha \times \beta$ is given as $\mathcal{S}(O, \mathscr{M})=\mathscr{V}(O)+\mathscr{V}(\mathscr{M})$ and $\mathcal{S}(O, \mathscr{M})=\left[\mathcal{S}_{i j}\right]$, where $\mathcal{S}_{i j}=\mathscr{V}_{i j}^{O}+\mathscr{V}_{i j}^{\mathscr{M}}$. The total score of each object in the universal set is $\left|\sum_{j=1}^{n} \mathcal{S}_{i j}\right|$.

\section{A Decision-Making Approach for Neutrosophic Hypersoft Matrix Using Score Function}

A set of decision-makers wants to choose the appropriate alternative from $\alpha$ number of alternatives. Decision-makers select the attributes $(\beta)$ for the selection of the most suitable alternative. If anyone attribute has further subattributes which form a relation like NHSM, every decision-maker gives his preference for each alternative according to the subattributes of the considered attributes in form of NHSMs and obtained an NHSM of order $\alpha \times \beta$. From this NHSM, we calculate values of matrices, which help to obtain a scoring matrix, and finally, we calculate the total score of each alternative from the score matrix. The value matrices are matrices that obey all properties of the real matrices. The score function is also a real matrix that is obtained from two or more value matrices. The algorithm of the presented approach is given as follows.

5.1. Algorithm. Step 1: construct an NHSM.

Step 2: calculate the value matrix from NHSM. Let $O=$ $\left[O_{i j}\right]$ be the NHSM of order $\alpha \times \beta$, where $O_{i j}=\left(\mathscr{T}_{i j k}^{o}, \mathscr{I}_{i j k}^{o}, \mathscr{F}_{i j k}^{o}\right)$, then the value of matrix $O$ is denoted as $\mathscr{V}(O)$ and it is defined as $\mathscr{V}(O)=\left[\mathscr{V}_{i j}^{O}\right]$ of order $\alpha \times \beta$, where $\mathscr{V}_{i j}^{O}=\mathscr{T}_{i j k}^{o}-\mathscr{I}_{i j k}^{o},-\mathscr{F}_{i j k}^{o}$.

Step 3: compute score matrix with the help of value matrices. The score of two NHSMs $O=\left[O_{i j}\right]$ and $\mathscr{M}=$ $\left[\mathscr{M}_{i j}\right]$ of order $\alpha \times \beta$ is given as $\mathcal{S}(O, \mathscr{M})=\mathscr{V}(O)+$ $\mathscr{V}(\mathscr{M})$ and $\mathcal{S}(O, \mathscr{M})=\left[\mathcal{S}_{i j}\right]$, where $\mathcal{S}_{i j}=\mathscr{V}_{i j}^{O}+\mathscr{V}_{i j}^{\mathscr{M}}$. Step 4: compute the total score from the score matrix. The total score of each object in the universal set is $\left|\sum_{j=1}^{n} \mathcal{S}_{i j}\right|$.

Step 5: find the optimal solution by selecting an object of maximum score from the total score matrix.

5.2. Application of the Proposed Approach. Let $\mathcal{U}=\left\{\mathscr{T}^{1}, \mathscr{T}^{2}, \mathscr{T}^{3}, \mathscr{T}^{4}, \mathscr{T}^{5}, \mathscr{T}^{6}, \mathscr{T}^{7}, \mathscr{T}^{8}, \mathscr{T}^{9}, \mathscr{T}^{10}, \mathscr{T}^{11}, \mathscr{T}^{12}\right.$, $\left.\mathscr{T}^{13}, \mathscr{T}^{14}, \mathscr{T}^{15}\right\}$ be a collection of applicants for the position of a faculty member in the public sector. The president of the institution hires a team of decision-makers $\{\mathbb{A}, \mathbb{B}\}$ for the selection of the most competent faculty member. Also, the president instructs decision-makers about the selection procedure of the alternative such as

$$
\mathscr{A}=\left\{\mathscr{A}_{1}=\text { Qualification, } \mathscr{A}_{2}=\text { Experience, } \mathscr{A}_{3}=\text { Gender, } \mathscr{A}_{4}=\text { Publications }\right\} \text {. }
$$


Furthermore, the abovementioned attributes have further bifurcation and can be classified as follows:

$$
\begin{aligned}
& \mathscr{A}_{1}^{a}=\text { Qualification }=\left\{\text { BS Hons. }, \frac{\mathrm{MS}}{\mathrm{Mphill}}, \text { Phd, Post Doctorate }\right\}, \\
& \mathscr{A}_{2}^{b}=\text { Experience }=\{5 \mathrm{yr}, 8 \mathrm{yr}, 10 \mathrm{yr}, 15 \mathrm{yr}\}, \\
& \mathscr{A}_{3}^{c}=\text { Gender }=\{\text { Male, Female }\} \\
& \mathscr{A}_{4}^{d}=\text { Publications }=\{3,5,8,10+\} .
\end{aligned}
$$

Then,

$$
\mathscr{F}: \mathscr{A}_{1}^{a} \times \mathscr{A}_{2}^{b} \times \mathscr{A}_{3}^{c} \times \mathscr{A}_{4}^{d} \longrightarrow P(\mathcal{U}) .
$$

Assume the relation $\mathscr{F}\left(\mathscr{A}_{1}^{a} \times \mathscr{A}_{2}^{b} \times \mathscr{A}_{3}^{c} \times \mathscr{A}_{4}^{d}\right)=\mathscr{F}$ (Mphill, $5 \mathrm{yr}$, male, 3 ) is the actual requirement of the institute for the selection of the most competent candidate. On a seniority basis, four are shortlisted $\left\{\mathscr{T}^{2}, \mathscr{T}^{6}, \mathscr{T}^{8}, \mathscr{T}^{14}\right\}$ according to the above-defined relation (Mphill, $5 \mathrm{yr}$, male, 3$)$. The team of decision-makers $\{\mathbb{A}, \mathbb{B}\}$ interviews the shortlisted candidates. Both decision-makers give their opinion for each alternative in the form of NHSM given as follows:

$$
\begin{aligned}
& \mathbb{A}= \mathscr{F}(\text { Mphill, } 5 \text { yr, male }, 3) \\
&=\left\{\begin{array}{l}
\left(\mathscr{T}^{2},\{0.5,0.3,0.6\},\{0.3,0.4,0.7\},\{0.5,0.6,0.9\},\{0.6,0.4,0.5\}\right), \\
\left(\mathscr{T}^{6},\{0.3,0.2,0.1\},\{0.6,0.5,0.3\},\{0.7,0.8,0.3\},\{0.7,0.5,0.3\}\right), \\
\left(\mathscr{T}^{8},\{0.7,0.3,0.6\},\{0.6,0.4,0.8\},\{0.8,0.5,0.4\},\{0.6,0.2,0.1\}\right), \\
\left(\mathscr{T}^{14},\{0.5,0.4,0.5\},\{0.3,0.6,0.7\},\{0.9,0.2,0.1\},\{0.4,0.5,0.3\}\right)
\end{array}\right\}, \\
& \mathbb{B}=\mathscr{F}(\text { Mphill, } 5 \text { yr, male }, 3) \\
&=\left\{\begin{array}{l}
\left(\mathscr{T}^{2},\{0.8,0.1,0.2\},\{0.7,0.4,0.3\},\{0.4,0.6,0.3\},\{0.5,0.3,0.5\}\right), \\
\left(\mathscr{T}^{6},\{0.8,0.2,0.1\},\{0.7,0.4,0.3\},\{0.8,0.2,0.1\},\{0.9,0.3,0.2\}\right), \\
\left(\mathscr{T}^{8},\{0.5,0.3,0.4\},\{0.7,0.3,0.2\},\{0.9,0.2,0.1\},\{0.4,0.2,0.7\}\right), \\
\left(\mathscr{T}^{14},\{0.7,0.4,0.2\},\{0.2,0.4,0.7\},\{0.7,0.2,0.1\},\{0.6,0.3,0.4\}\right)
\end{array}\right\} .
\end{aligned}
$$

Utilize the above-developed algorithm such as follows:
Step 1. The above two NHSSs are given in the form of NHSMs as

$$
\begin{aligned}
& {[\mathbb{A}]=\left[\begin{array}{llll}
(0.5,0.3,0.6) & (0.3,0.4,0.7) & (0.5,0.6,0.9) & (0.6,0.4,0.5) \\
(0.3,0.2,0.1) & (0.6,0.5,0.3) & (0.7,0.8,0.3) & (0.7,0.5,0.3) \\
(0.7,0.3,0.6) & (0.6,0.4,0.8) & (0.8,0.5,0.4) & (0.6,0.2,0.1) \\
(0.5,0.4,0.5) & (0.3,0.6,0.7) & (0.9,0.2,0.1) & (0.4,0.5,0.3)
\end{array}\right],} \\
& {[\mathbb{B}]=\left[\begin{array}{llll}
(0.8,0.1,0.2) & (0.7,0.4,0.3) & (0.4,0.6,0.3) & (0.5,0.3,0.5) \\
(0.8,0.2,0.1) & (0.7,0.4,0.3) & (0.8,0.2,0.1) & (0.9,0.3,0.2) \\
(0.5,0.3,0.4) & (0.7,0.3,0.2) & (0.9,0.2,0.1) & (0.4,0.2,0.7) \\
(0.7,0.4,0.2) & (0.2,0.4,0.7) & (0.7,0.2,0.1) & (0.6,0.3,0.4)
\end{array}\right] .}
\end{aligned}
$$


TABLE 7: Comparative analysis of final ranking with existing studies.

\begin{tabular}{lcc}
\hline Methods & Ranking of alternatives & Optimal alternatives \\
\hline Cagman and Enginoglu [53] & $\mathscr{T}^{2}>\mathscr{T}^{14}>\mathscr{T}^{8}>\mathscr{T}^{6}$ & $\mathscr{T}^{2}$ \\
Cagman and Enginoglu [54] & $\mathscr{T}^{2}>\mathscr{T}^{8}>\mathscr{T}^{14}>\mathscr{T}^{6}$ & $\mathscr{T}^{2}$ \\
Mondal and Roy [57] & $\mathscr{T}^{2}>\mathscr{T}^{14}>\mathscr{T}^{8}>\mathscr{T}^{6}$ & $\mathscr{T}^{2}$ \\
Deli and Broumi [59] & $\mathscr{T}^{2}>\mathscr{T}^{14}>\mathscr{T}^{6}>\mathscr{T}^{8}$ & $\mathscr{T}^{2}$ \\
Proposed approach & $\mathscr{T}^{2}>\mathscr{T}^{14}>\mathscr{T}^{8}>\mathscr{T}^{6}$ & $\mathscr{T}^{2}$ \\
\hline
\end{tabular}

Step 2. Now, calculate the value matrices of NHSMs defined in step I.

$$
\begin{aligned}
& {[\mathscr{V}(\mathbb{A})]=\left[\begin{array}{cccc}
-0.4 & -0.8 & -1 & -0.3 \\
0 & -0.2 & -0.4 & -0.1 \\
-0.2 & -0.6 & -0.1 & -0.3 \\
-0.4 & -1 & -.6 & -0.4
\end{array}\right],} \\
& {[\mathscr{V}(\mathbb{B})]=\left[\begin{array}{cccc}
0.5 & 0 & -0.5 & -0.3 \\
0.5 & 0 & 0.5 & 0.4 \\
-0.2 & 0.2 & 0.6 & 0.5 \\
0.1 & -0.9 & 0.4 & -0.1
\end{array}\right] .}
\end{aligned}
$$

Step 3. Now, compute the score matrix by adding value matrices obtained in step II.

$$
[\mathcal{S}(\mathbb{A}, \mathbb{B})]=\left[\begin{array}{cccc}
0.1 & -0.8 & -1.5 & -0.6 \\
0.5 & -0.2 & 0.1 & 0.3 \\
-0.4 & -0.4 & 0.5 & -0.2 \\
-0.3 & -1.9 & 1 & -0.5
\end{array}\right] .
$$

Step 4. Now, a total score of score matrix is given as

$$
\text { Total score }=\left[\begin{array}{l}
2.8 \\
0.1 \\
0.5 \\
1.7
\end{array}\right]
$$

Step 5. The candidate $\mathscr{T}^{2}$ will be selected for a faculty position in the public sector as the total score of $\mathscr{T}^{2}$ is highest among the rest of the total score of candidates.

\section{Discussion and Comparative Analysis}

In the subsequent section, we are going to compare and discuss our proposed decision-making methodology with some existing techniques.

6.1. Comparative Analysis. Through the current exploration along with comparative, it can be concluded that the results acquired by the proposed approach overlap with the accessible methodologies. However, in connection with accessible decision-making techniques, the main benefit of the proposed method is that it comprises additional information utilizing the membership, nonmembership, and indeterminacy of subattributes of considered attributes to address uncertainty in data. Among them, the information related to the object can be interpreted more accurately and objectively. It is also a useful tool for solving inaccurate and imprecise data in the DM process. Therefore, the inspiration for the score value corresponding to each parameter will not affect other parameters, so predictable information loss will occur in the process. On the contrary, there is no serious loss of information in our proposed approach. The benefit of the planned method along with related measures over existing methods is that it not just notices the degree of discrimination, but the degree of similarity between observations so that avoiding decisions is based on negative reasons. Therefore, it is a suitable tool to combine inaccurate and uncertain info in the DM process.

6.2. Discussion. By utilizing the methodology of Cagman and Enginoglu [53], we can process the parametric values of the alternative, but soft matrices have no information about the multi-subattributes of the considered parameters. Cagman and Enginoglu [54] deal with the uncertainty considering MD, but the fuzzy soft matrix cannot deliver the NMD of any attribute of the alternative. To overcome such difficulties, Mondal and Roy [57] utilized the intuitionistic fuzzy soft matrices using MD and NMD of the parametrized family of attributes, but intuitionistic fuzzy soft matrices provide no information about the indeterminacy part of the object. Deli and Broumi [59] established neutrosophic soft matrices competently handle the truthiness, indeterminacy, and falsity objects of the attributes, but all the abovementioned studies have no information about the subattributes of the considered attributes, when attributes have their corresponding subattributes, then all abovementioned theories fail to handle the situation. On the contrary, our presented notion competently deals with such difficulties considering the truthness, indeterminacy, and falsity objects of any subattribute of the considered parameters with the following condition $0 \leq \mathscr{T}(v)+\mathscr{I}(v)+\mathscr{F}(v) \leq 3$. Consequently, relying upon the obtained results, it can be confidently concluded that the proposed methodology indicates a higher stability and usability for decision-makers in the DM procedure. The presented NHSM is unable to deal with the situation, when the information is delivered in interval form. The final ranking of the proposed technique and prevailing methodologies is seen in Table 7.

\section{Conclusion}

In this paper, we extend the notion of NHSS to a neutrosophic hypersoft matrix with some basic operations and discussed their desirable properties. We also proposed the 
score matrix for NHSM and furthermore introduced the logical operators, necessity, and possibility operators under considered environment. A decision-making methodology has been established with the assistance of the score matrix to solve the MADM problem. Consequently, relying upon the obtained outcomes, it can be confidently concluded that the proposed methodology indicates a greater constancy and usability for decision-makers in the DM process. To ensure the practicality of the established approach, a comparative study has been conducted. Future research will surely concentrate upon presenting several other operators to solve decision-making complications utilizing NHSM. The suggested idea can be applied in quite a lot of issues in real life, including the medical profession, pattern recognition, and economics. We are sure this article will open new vistas for investigators in this field.

\section{Data Availability}

No such type of data is used in this research.

\section{Conflicts of Interest}

The authors declare that there are no conflicts of interest.

\section{Authors' Contributions}

Rana Muhammad Zulqarnain, Fahd Jarad, Thabet Abdeljawad, and Abdul Samad contributed equally to this work.

\section{Acknowledgments}

The author Rifaqat Ali would like to express his gratitude to Deanship of Scientific Research at King Khalid University, Saudi Arabia, for funding research groups under the research grant number R.G.P. 1/162/42.

\section{References}

[1] L. A. Zadeh, "Fuzzy sets," Information and Control, vol. 8, no. 3, pp. 338-353, 1965.

[2] K. T. Atanassov, "Intuitionistic fuzzy sets," Fuzzy Sets and Systems, vol. 20, no. 1, pp. 87-96, 1986.

[3] R. R. Yager, "Pythagorean fuzzy subsets," in Proceedings Joint IFSA World Congress and NAFIPS Annual Meeting, pp. 57-61, Edmonton, Canada, 2013.

[4] R. R. Yager, "Pythagorean membership grades in multicriteria decision making," IEEE Transactions on Fuzzy Systems, vol. 22, no. 4, pp. 958-965, 2014.

[5] X. Zhang and Z. Xu, "Extension of TOPSIS to multiple criteria decision making with pythagorean fuzzy sets," International Journal of Intelligent Systems, vol. 29, no. 12, pp. 1061-1078, 2014.

[6] G. Wei and M. Lu, "Pythagorean fuzzy power aggregation operators in multiple attribute decision making," International Journal of Intelligent Systems, vol. 33, no. 1, pp. 169-186, 2018.

[7] L. Wang and N. Li, "Pythagorean fuzzy interaction power bonferroni mean aggregation operators in multiple attribute decision making," International Journal of Intelligent Systems, vol. 35, no. 1, pp. 150-183, 2020.
[8] H. Gao, M. Lu, G. Wei, and Y. Wei, "Some novel pythagorean fuzzy interaction aggregation operators in multiple attribute decision making," Fundamenta Informaticae, vol. 159, no. 4, pp. 385-428, 2018.

[9] G. Wei, "Pythagorean fuzzy interaction aggregation operators and their application to multiple attribute decision making," Journal of Intelligent \& Fuzzy Systems, vol. 33, no. 4, pp. 2119-2132, 2017.

[10] X. Zhang, "A novel approach based on similarity measure for pythagorean fuzzy multiple criteria group decision making," International Journal of Intelligent Systems, vol. 31, no. 6, pp. 593-611, 2016.

[11] L. Wang, H. Garg, and N. Li, "Pythagorean fuzzy interactive hamacher power aggregation operators for assessment of express service quality with entropy weight," Soft Computing, pp. 1-21, 2020.

[12] L. Wang and N. Li, "Continuous interval-valued Pythagorean fuzzy aggregation operators for multiple attribute group decision making," Journal of Intelligent \& Fuzzy Systems, vol. 36, no. 6, pp. 6245-6263, 2019.

[13] X. Peng and H. Yuan, "Fundamental properties of pythagorean fuzzy aggregation operators," Fundamenta Informaticae, vol. 147, no. 4, pp. 415-446, 2016.

[14] X. Peng and Y. Yang, "Some results for pythagorean fuzzy sets," International Journal of Intelligent Systems, vol. 30, no. 11, pp. 1133-1160, 2015.

[15] H. Garg, "New logarithmic operational laws and their aggregation operators for pythagorean fuzzy set and their applications," International Journal of Intelligent Systems, vol. 34, no. 1, pp. 82-106, 2019.

[16] R. Arora and H. Garg, "Group decision-making method based on prioritized linguistic intuitionistic fuzzy aggregation operators and its fundamental properties," Computational and Applied Mathematics, vol. 38, no. 2, pp. 1-36, 2019.

[17] Z. Ma and Z. Xu, "Symmetric pythagorean fuzzy weighted geometric/averaging operators and their application in multicriteria decision-making problems," International Journal of Intelligent Systems, vol. 31, no. 12, pp. 1198-1219, 2016.

[18] D. Molodtsov, "Soft set theory-First results," Computers \& Mathematics with Applications, vol. 37, no. 4-5, pp. 19-31, 1999.

[19] P. K. Maji, R. Biswas, and A. R. Roy, "Soft set theory," Computers \& Mathematics with Applications, vol. 45, no. 4-5, pp. 555-562, 2003.

[20] P. K. Maji, A. R. Roy, and R. Biswas, "An application of soft sets in a decision making problem," Computers \& Mathematics with Applications, vol. 44, no. 8-9, pp. 1077-1083, 2002.

[21] P. K. Maji, R. Biswas, and A. R. Roy, "Fuzzy soft sets," Journal of Fuzzy Mathematics, vol. 9, pp. 589-602, 2001.

[22] P. K. Maji, R. Biswas, and A. R. Roy, "Intuitionistic fuzzy soft sets," Journal of Fuzzy Mathematics, vol. 9, pp. 677-692, 2001.

[23] H. Garg and R. Arora, "Generalized and group-based generalized intuitionistic fuzzy soft sets with applications in decision-making," Applied Intelligence, vol. 48, no. 2, pp. 343-356, 2018.

[24] H. Garg, R. Arora, and R. Arora, "TOPSIS method based on correlation coefficient for solving decision-making problems with intuitionistic fuzzy soft set information," AIMS Mathematics, vol. 5, no. 4, pp. 2944-2966, 2020.

[25] R. M. Zulqarnain, X. L. Xin, M. Saqlain, and W. A. Khan, "TOPSIS method based on the correlation coefficient of interval-valued intuitionistic fuzzy soft sets and aggregation 
operators with their application in decision-making," Journal of Mathematics, vol. 2021, Article ID 6656858, 16 pages, 2021.

[26] X. Peng, Y. Yang, and J. Song, "Pythagoren fuzzy soft set and its application," Computer Engineering, vol. 41, no. 7, pp. 224-229, 2015.

[27] T. M. Athira, S. J. John, and H. Garg, "A novel entropy measure of pythagorean fuzzy soft sets," AIMS Mathematics, vol. 5, no. 2, pp. 1050-1061, 2020.

[28] T. M. Athira, S. J. John, and H. Garg, "Entropy and distance measures of pythagorean fuzzy soft sets and their applications," Journal of Intelligent \& Fuzzy Systems, vol. 37, no. 3, pp. 4071-4084, 2019.

[29] R. M. Zulqarnain, X. L. Xin, H. Garg, and W. A. Khan, "Aggregation operators of pythagorean fuzzy soft sets with their application for green supplier chain management," Journal of Intelligent \& Fuzzy Systems, vol. 40, no. 3, pp. 5545-5563, 2021.

[30] M. Riaz, K. Naeem, and D. Afzal, "Pythagorean m-polar fuzzy soft sets with TOPSIS method for MCGDM," Punjab University Journal of Mathematics, vol. 52, no. 3, pp. 21-46, 2020.

[31] M. Riaz, N. Khalid, and D. Afzal, "A similarity measure under pythagorean fuzzy soft environment with applications," Computational and Applied Mathematics, vol. 39, no. 4, pp. 1-17, 2020.

[32] Q. Han, W. Li, Y. Song, T. Zhang, and R. Wang, "A new method for MAGDM based on improved TOPSIS and a novel pythagorean fuzzy soft entropy," Symmetry, vol. 11, no. 7, p. 905, 2019.

[33] R. M. Zulqarnain, X. L. Xin, I. Siddique, W. Asghar Khan, and M. A. Yousif, "TOPSIS method based on correlation coefficient under pythagorean fuzzy soft environment and its application towards green supply chain management," Sustainability, vol. 13, no. 4, p. 1642, 2021.

[34] F. Smarandache, Neutrosophy: Neutrosophic Probability, Set, and Logic: Analytic Synthesis \& Synthetic Analysis, Rehoboth Press, Coimbatore, India, 1998.

[35] P. K. Maji, "Neutrosophic soft set," Annals of Fuzzy Mathematics and Informatics, vol. 5, no. 1, pp. 157-168, 2013.

[36] F. Karaaslan, "Possibility neutrosophic soft sets and PNSdecision making method," Applied Soft Computing Journal, vol. 54, pp. 403-414, 2016.

[37] S. Broumi, "Generalized neutrosophic soft set," International Journal of Computer Science, Engineering and Information Technology, vol. 3, no. 2, pp. 17-30, 2013.

[38] I. Deli and Y. Şubaş, "A ranking method of single valued neutrosophic numbers and its applications to multi-attribute decision making problems," International Journal of Machine Learning and Cybernetics, vol. 8, no. 4, pp. 1309-1322, 2017.

[39] H. Wang, F. Smarandache, and Y. Zhang, "Single valued neutrosophic sets," International Journal of General Systems, vol. 42, pp. 386-394, 2013.

[40] J. Ye, "A multicriteria decision-making method using aggregation operators for simplified neutrosophic sets," Journal of Intelligent \& Fuzzy Systems, vol. 26, no. 5, pp. 2459-2466, 2014.

[41] M. R. Hashmi, M. Riaz, and F. Smarandache, "m-Polar neutrosophic topology with applications to multi criteria decision-making in medical diagnosis and clustering analysis," International Journal of Fuzzy Systems, 2019.

[42] R. M. Zulqarnain, X. L. Xin, M. Saeed, F. Smarandache, and N. Ahmad, "Generalized neutrosophic TOPSIS to solve multicriteria decision-making problems," Neutrosophic Sets and Systems, vol. 38, pp. 276-292, 2020.
[43] R. M. Zulqarnain, X. L. Xin, M. Saqlain, F. Smarandache, and M. I. Ahamad, "An integrated model of neutrosophic TOPSIS with application in multi-criteria decision-making problem," Neutrosophic Sets, and Systems, vol. 40, pp. 118-133, 2021.

[44] F. Smarandache, "Extension of soft set to hypersoft set, and then to plithogenic hypersoft set," Neutrosophic Sets and Systems, vol. 22, pp. 168-170, 2018.

[45] S. Rana, M. Qayyum, M. Saeed, and F. Smarandache, "Plithogenic fuzzy whole hypersoft set: construction of operators and their application in frequency matrix multi attribute decision making technique," Neutrosophic Sets and Systems, vol. 28, pp. 34-50, 2019.

[46] R. M. Zulqarnain, X. L. Xin, M. Saqlain, and F. Smarandache, "Generalized aggregate operators on neutrosophic hypersoft set," Neutrosophic Sets and Systems, vol. 36, pp. 271-281, 2020.

[47] R. M. Zulqarnain, X. L. Xin, and M. Saeed, "Extension of TOPSIS method under intuitionistic fuzzy hypersoft environment based on correlation coefficient and aggregation operators to solve decision making problem," AIMS Mathematics, vol. 6, no. 3, pp. 2732-2755, 2020.

[48] R. M. Zulqarnain, X. L. Xin, and M. Saeed, A Development of Pythagorean Fuzzy Hypersoft Set with Basic Operations and Decision-Making Approach Based on the Correlation Coefficient, Theory and Application of Hypersoft Set, pp. 85-106, Pons Publishing House Brussels, Rotterdam, Netherlands, 2021.

[49] R. M. Zulqarnain, I. Saddique, F. Jarad, R. Ali, and T. Abdeljawad, "Development of TOPSIS technique under pythagorean fuzzy hypersoft environment based on correlation coefficient and its application towards the selection of antivirus mask in COVID-19 pandemic," Complexity, vol. 2021, Article ID 6634991, 27 pages.

[50] A. Samad, R. M. Zulqarnain, E. Sermutlu et al., "Selection of an effective hand sanitizer to reduce covid-19 effects and extension of topsis technique based on correlation coefficient under neutrosophic hypersoft set," Complexity, Article ID 5531830, 19 pages.

[51] R. M. Zulqarnain, X. L. Xin, M. Saqlain, M. Saeed, F. Smarandache, and M. I. Ahamad, "Some fundamental operations on interval valued neutrosophic hypersoft set with their properties," Neutrosophic Sets and Systems, vol. 40, pp. 134-148, 2021.

[52] R. M. Zulqarnain, X. L. Xin, B. Ali, S. Broumi, S. Abdal, and M. I. Ahamad, "Decision-making approach based on correlation coefficient with its properties under interval-valued neutrosophic hypersoft set environment," Neutrosophic Sets and Systems, vol. 40, pp. 12-28, 2021.

[53] N. Cagman and S. Enginoglu, "Soft matrix theory and its decision making," Computers and Mathematics with Applications, vol. 59, pp. 3308-3314, 2010.

[54] N. Cagman and S. Enginolu, "Fuzzy soft matrix theory and its applications in decision making," Iranian Journal of Fuzzy Systems, vol. 9/1, pp. 109-119, 2012.

[55] B. K. Saikia, H. Boruah, and H. Boruah, "An appliaction of generalized fuzzy soft matrices in decision making problem," IOSR Journal of Mathematics, vol. 10, no. 1, pp. 33-41, 2014.

[56] S. Broumi, F. Smarandache, and M. Dhar, "On fuzzy soft matrix based on reference function," International Journal of Information Engineering and Electronic Business, vol. 5, no. 2, pp. 52-59, 2013.

[57] J. I. Mondal and T. K. Roy, "Some properties on intuitionistic fuzzy soft matrices," International Journal of Mathematics Research, vol. 5/2, pp. 267-276, 2013. 
[58] A. Kalaichelvi and P. Kanimozhi, "Impact of excessve television Viewing by children an analysis using intuitionistic fuzzy soft Matrces," Int Jr. Of Mathematics Sciences and Applications, vol. 3/1, pp. 103-108, 2013.

[59] I. Deli and S. Broumi, "Neutrosophic soft matrices and NSMdecision making," Journal of Intelligent \& Fuzzy Systems, vol. 28, no. 5, pp. 2233-2241, 2015. 